EPJ manuscript No.

(will be inserted by the editor)

\title{
Gap and out-gap breathers in a binary modulated discrete nonlinear Schrödinger model
}

\author{
Andrey V. Gorbach and Magnus Johansson \\ Department of Physics and Measurement Technology (IFM), \\ . Linköping University, S-581 83 Linköping, Sweden, e-mail: mjn@ifm.liu.se \\ Received: date / Revised version: date
}

\begin{abstract}
We consider a modulated discrete nonlinear Schrödinger (DNLS) model with alternating on-site potential, having a linear spectrum with two branches separated by a 'forbidden' gap. Nonlinear localized time-periodic solutions with frequencies in the gap and near the gap - discrete gap and out-gap breathers (DGBs and DOGBs) - are investigated. Their linear stability is studied varying the system parameters from the continuous to the anti-continuous limit, and different types of oscillatory and real instabilities are revealed. It is shown, that generally DGBs in infinite modulated DNLS chains with hard (soft) nonlinearity do not possess any oscillatory instabilities for breather frequencies in the lower (upper) half of the gap. Regimes of 'exchange of stability' between symmetric and antisymmetric DGBs are observed, where an increased breather mobility is expected. The transformation from DGBs to DOGBs when the breather frequency enters the linear spectrum is studied, and the general bifurcation picture for DOGBs with tails of different wave numbers is described. Close to the anti-continuous limit, the localized linear eigenmodes and their corresponding eigenfrequencies are calculated analytically for several gap/out-gap breather configurations, yielding explicit proof of their linear stability or instability close to this limit.
\end{abstract}

PACS. 63.20.Pw Localized modes - 63.20.Ry Anharmonic lattice modes - 42.65 Nonlinear waveguides

\section{Introduction}

The existence of spatially localized nonlinear excitations in discrete models - 'discrete breathers' (DBs) - has attracted much attention, as they can play a significant role in condensed matter physics, biophysics, nonlinear optics, etc. (for reviews see e.g. 1,2]). In particular, being localized in space and typically stable, DBs can contribute to energy and information transfer processes. For example, breathers can trap energy during long periods of time and cause non-exponential thermal relaxation in nonlinear lattices 3]. By contrast, under certain conditions 4,5] DBs can be rather mobile and become good energy carriers.

The presence of intrinsic structure of a medium can influence the DB properties. In particular, new types of DBs - discrete gap breathers (DGBs) - appear with frequencies inside the forbidden gaps in the linear waves spectrum. DGBs are the discrete analogues of gap solitons, first discovered in a nonlinear optical medium with modulated refractive index [6]. Their specific features are due to the existence of two neighboring bands of the linear dispersion curve, with opposite signs of dispersion close to a gap.

A classical example of a system with two bands in the linear spectrum is a diatomic one-dimensional lattice. DGBs in diatomic lattices with nonlinear interatomic potentials [Fermi-Pasta-Ulam (FPU) models] were studied analytically as well as numerically within the rotating- wave approximation, neglecting higher harmonics generation 1,8, 9, 10,11. Later the existence of DGBs in diatomic FPU lattices was proved rigorously [12,13. Existence and linear stability properties of DGBs in diatomic FPU chains were also studied numerically for some particular values of system parameters [14,15,16]. We recently 17. performed a detailed analysis of DGBs in a diatomic lattice with nonlinear on-site potential - Klein-Gordon (KG) model - in the complete regime of continuation from the anti-continuous (AC) to the continuous limit, and in particular we described the dynamics resulting from several types of instability mechanisms (oscillatory as well as non-oscillatory).

Very recently, much attention has been attracted to modulated structures described by equations of discrete nonlinear Schrödinger (DNLS) type, in particular within nonlinear optics for the description of electromagnetic waves in arrays of weakly coupled optical waveguides [18. For such a system with even and odd waveguides of different widths, discrete gap [19,20] as well as multigap [21] solitons were found. A related model with two coupled DNLSlike equations describing a diffraction-managed waveguide array was also studied recently, and found to support different types of discrete gap solitons 22. Much recent attention has also been given to DNLS models in describing Bose-Einstein condensates (BECs) trapped in optical pe- 
A.V. Gorbach, M. Johansson: Gap and out-gap breathers in a binary modulated DNLS model

riodic potentials 23 24. For a recent review on properties and applications of the DNLS equations, see [25].

As the DNLS equations also approximate the smallamplitude dynamics of weakly coupled KG-chains [26,27, the DNLS discrete gap solitons should share many features of DGBs in KG (and also FPU) lattices (henceforth they will therefore also be referred to as DGBs). In particular, in the continuum limit these models are described by the same general class of coupled equations with exact gap soliton solutions analyzed e.g. in 28 29.

The aim of the present study is to give a detailed and thorough analysis of DGB properties in the modulated DNLS model. Our motivation is twofold. First, we wish to provide, for the benefit e.g. of experimentalists working in related areas (nonlinear optics, BECs), charts of different types of instabilities etc., and information about the parameter regimes where they are expected to be seen. Particularly interesting is the possible observation of moving DGBs. Generally DBs can not freely propagate along the system due to the existence of the effective periodic Peierls-Nabarro (PN) potential (caused by the discreteness of the system). However, in the diatomic KG model we found 17] parameter regimes with increased DGB mobility due to 'exchange of stability' between symmetric and antisymmetric DGB configurations, and thus it is of interest to look for similar effects in the modulated DNLS models, proposing that the increased DGB mobility should be directly observable in experiments.

Second, it was not carefully analyzed before, within a fully discrete model, what happens with DGBs when their frequencies approach gap boundaries. In continuous models, gap solitons delocalize and vanish at one gap boundary, but bifurcate into a new type of excitations - outgap solitons - at the other boundary [30. Out-gap solitons have frequencies inside the linear spectrum, and their structure can be viewed as a superposition of two fields. One field has a 'dark' soliton shape with non-zero amplitudes at the infinities and a localized decrease in the center. The other field has either a 'bright' soliton form with tails exponentially decaying to zero, or forms a 'bright soliton on a pedestal' ('anti-dark soliton'), similar to a 'dark' soliton but with a localized increase of the amplitude in the center. Although out-gap solitons can radiate energy through linear waves, they may still represent long-lived localized excitations persistent to perturbations (e.g. 31]).

Thus, analogously it is interesting to look for discrete out-gap breathers (DOGBs) in discrete models, and to study their stability and the bifurcations from DGBs to DOGBs. This was not analyzed in earlier studies of DGBs in KG or FPU models, partly due to technical problems: since DGBs delocalize when approaching gap boundaries, numerical computations become time consuming when increasing the system size to avoid boundary effects. However, DNLS models are more conveniently analyzed numerically, since time-periodic DGBs become 'stationary' solutions with purely harmonic time-dependence, that can be removed by transforming into a rotating frame.

Another important question concerns the possible existence of truly localized excitations with frequencies inside the linear wave spectrum - analogues of 'embedded' solitons (e.g. [32]) - and the nature of tails in such hypothetical localized DOGBs. This issue is also most conveniently analyzed with the modulated DNLS model.

Our study of the spatially binary modulated DNLS model and the properties of its DGB and DOGB solutions will be structured as follows. In Sec. 2 the model is described, as well as the properties of its linear spectrum. In Sec. 3 we discuss the linear stability analysis of stationary solutions, and in Sec. 4 4 we review the numerical construction of breather solutions from the anti-continuous limit, discussing some particular features of the continuation in coupling and frequency of DGBs and DOGBs. In Sec. 5 we present numerical and analytical results for linear stability properties of different types of DGBs and DOGBs. Sec.5.1 describes the continuation in coupling of DGBs, and we compare the stability results with those obtained for the diatomic KG chain [17. In Sec. 5.2 we study the transition from DGBs into DOGBs while changing the breather frequency, describing the bifurcations which may occur to DGBs when their frequency approach the gap boundary, and furthermore penetrate the linear spectrum as DOGBs. In Sec. 5.3 bifurcations of DOGBs with different types of tails are described. We study the subsequent bifurcations of DOGBs into 'on-top' DBs (DOTBs) with frequencies above the linear spectrum, and obtain a general bifurcation picture of DOGBs with tails of different wave numbers. We discuss the linear stability properties of the most important DOGB and DOTB solutions in Sec. 5.4 and in Sec. [6] conclusions are made. Some details of the analytical investigation of linear stability properties of DGBs and DOGBs at small coupling are deferred to Appendix A

\section{The Model}

The standard DNLS equation with cubic nonlinearity reads:

$$
i \frac{d \psi_{n}}{d t}=\lambda \psi_{n}-C\left(\psi_{n-1}+\psi_{n+1}-2 \psi_{n}\right)+\gamma\left|\psi_{n}\right|^{2} \psi_{n} .
$$

When modelling an array of weakly coupled optical waveguides 18 19, $\psi_{n}$ is the normalized amplitude of the electric field in the EM wave, propagating along the array. The coefficient $\lambda$ then characterizes the linear propagation constant in the waveguide, $C$ the coupling coefficient, $\gamma$ the effective nonlinear coefficient, and the time-like variable $t$ measures distance along the array. Here we consider positive $\gamma$, corresponding to 'hard' nonlinearity. The case of 'soft' nonlinearity $(\gamma<0)$ can easily be recovered by a simple transformation, see footnote 2 below.

To introduce a gap parameter, one can modulate any of the constants $\lambda, C, \gamma$ (or two or three of them simultaneously). We choose to modulate the coefficient $\lambda$, corresponding in the optical model to a variation of the width of even and odd waveguides (see e.g. [19]). Thus, we put:

$$
\lambda \rightarrow \lambda_{n}=1+(-1)^{n} \delta^{2},
$$

where the parameter $\delta$ defines the width $\Delta$ of the gap in the linear waves spectrum: $\Delta=2 \delta^{2}$ (see below). 


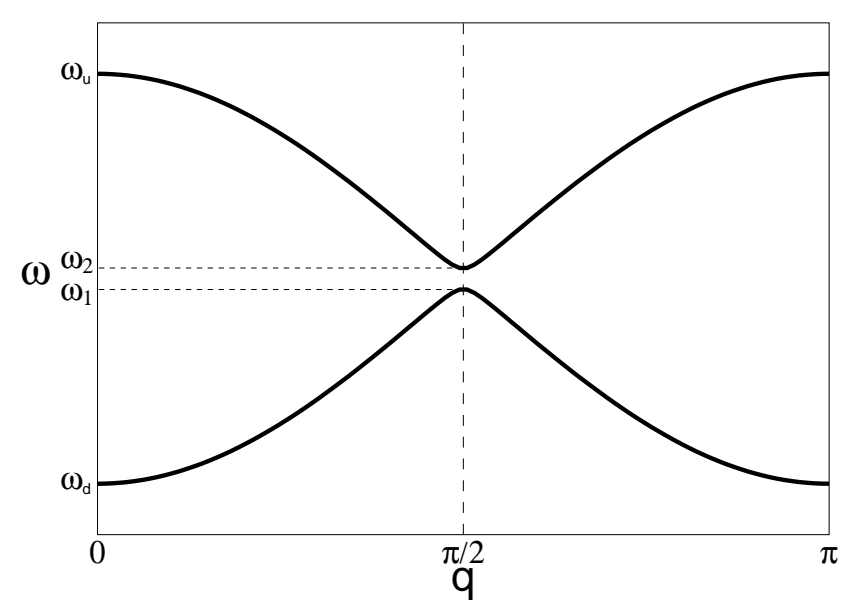

Fig. 1. The dispersion relation of linear waves (4).

The dispersion relation for solutions:

$$
\begin{aligned}
& \psi_{n}= \begin{cases}A \exp (i q n-i \omega t), & n=2 k, \\
B \exp (i q n-i \omega t), & n=2 k+1,\end{cases} \\
& k=0,1,2, \ldots
\end{aligned}
$$

of the linearized system (11) with $\lambda$ in the form (2) reads:

$$
\omega_{o, a}=1+2 C \pm \sqrt{\delta^{4}+4 C^{2} \cos ^{2}(q)},
$$

where indices $o$ and $a$ stand for the upper ('optic-like') and lower ('acoustic-like') branches of the spectrum ${ }^{1}$. It possesses a gap at the wave number $q=\pi / 2$ (see Fig. 1). The gap boundaries are defined as:

$$
\omega_{2,1}=1+2 C \pm \delta^{2}
$$

The ratio of the amplitudes $A$ and $B$ in a linear wave (3) is defined by its frequency $\omega$ :

$$
\frac{A}{B}=\sqrt{\left|\frac{\omega-\omega_{1}}{\omega-\omega_{2}}\right|} .
$$

In linear waves with frequencies belonging to the upper branch of the spectrum $\left(\omega>\omega_{2}\right)$, amplitudes in even waveguides $(A)$ are always higher than in odd waveguides $(B)$, while for frequencies in the lower branch $\left(\omega<\omega_{1}\right)$ the odd waveguides are dominant. Therefore, the sub-field of even (odd) waveguide amplitudes can be referred to as the upper (lower) band sub-field, respectively. With an analogy to a diatomic chain, even and odd waveguides correspond to light and heavy atoms, and will therefore be referred to as 'light' and 'heavy' sites, respectively.

Another type of modulated DNLS model is obtained by multiplying the left-hand side of equation (1) with $m_{n}$, defined analogously to $\lambda_{n}$ in (2). This model, corresponding to simultaneous modulation of all constants in (1), can be considered as a simplified model of a diatomic lattice

\footnotetext{
1 Note however that also for the "acoustic-like" branch the dispersion is quadratic and not linear for small $q$.
}

$\left(m_{n}\right.$ playing the role of atom masses), neglecting higher harmonics generation. As shown in 33. for the diatomic FPU model, the effect of higher harmonics on DB properties is often rather negligible, although additional instabilities and bifurcations generally may appear for large values of the coupling and/or large-amplitude oscillations (e.g. 27]). Investigating numerically breather properties in the two modulated DNLS models we have found qualitatively similar results, and therefore we focus our discussion only on model (11) with coefficients $\lambda$ as defined in (2).

We denote the system size (the number of waveguides) with $N(n=1,2, \ldots, N)$ and impose periodic boundary conditions $\psi_{N+1} \equiv \psi_{1}, \psi_{0} \equiv \psi_{N}$. The DNLS equations (11) are then the Hamiltonian equations with the Hamiltonian:

$$
\begin{aligned}
& H\left(\left\{i \psi_{n}\right\},\left\{\psi_{n}^{*}\right\}\right)= \\
& \quad \sum_{n=1}^{N}\left(C\left|\psi_{n+1}-\psi_{n}\right|^{2}+\lambda\left|\psi_{n}\right|^{2}+\frac{\gamma}{2}\left|\psi_{n}\right|^{4}\right) .
\end{aligned}
$$

In what follows we put $\gamma=+1$ in (1), (7) (without loss of generality, since varying $\gamma$ is equivalent to rescaling $\psi_{n}$ ).

\section{Stationary solutions and linear stability}

Stationary solutions of equations (11) have the form:

$$
\psi_{n}(t)=\phi_{n} e^{-i \omega_{b} t}
$$

with time-independent amplitudes $\phi_{n}$, where $\omega_{b}$ is the excitation frequency. DGBs have frequencies $\omega_{b}$ lying inside the gap (5), so that $\omega_{1}<\omega_{b}<\omega_{2}$. For DOGBs one has $\omega_{b}>\omega_{2}$, while 'on-top' breathers (DOTBs) have frequencies above the linear spectrum, $\omega_{b}>\omega_{u}$, where

$$
\omega_{u}=1+2 C+\sqrt{\delta^{4}+4 C^{2}} .
$$

To analyze the linear stability of a particular solution $\left\{\phi_{n}^{(0)}\right\}$, we add a small perturbation $\left\{\epsilon_{n}(t)\right\}$ to it:

$$
\psi_{n}(t)=\left(\phi_{n}^{(0)}+\epsilon_{n}(t)\right) e^{-i \omega_{b} t}
$$

and linearize the equations (11):

$$
\begin{aligned}
i \frac{d \epsilon_{n}}{d t}= & \left(\lambda_{n}+2 C-\omega_{b}\right) \epsilon_{n}-C\left(\epsilon_{n-1}+\epsilon_{n+1}\right)+ \\
& +2 \gamma\left|\phi_{n}^{(0)}\right|^{2} \epsilon_{n}+\gamma\left(\phi_{n}^{(0)}\right)^{2} \epsilon_{n}^{*}
\end{aligned}
$$

Expanding $\epsilon_{n}(t)$ in real and imaginary parts, $\epsilon_{n}(t)=\alpha_{n}(t)+$ $i \beta_{n}(t)$, equations (11) can, for real solutions $\left\{\phi_{n}^{(0)}\right\}$, be written in matrix form 34]

$$
\left(\begin{array}{c}
\left\{\dot{\alpha}_{n}\right\} \\
\left\{\dot{\beta}_{n}\right\}
\end{array}\right)=\left(\begin{array}{cc}
0 & \mathcal{L}_{0} \\
-\mathcal{L}_{1} & 0
\end{array}\right)\left(\begin{array}{l}
\left\{\alpha_{n}\right\} \\
\left\{\beta_{n}\right\}
\end{array}\right)
$$

with $\mathcal{L}_{0}$ and $\mathcal{L}_{1}$ defined as follows:

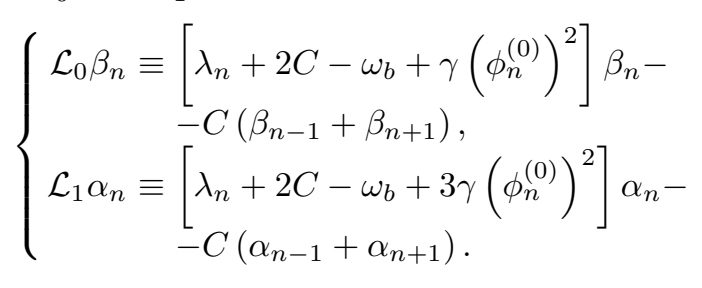


The solution $\left\{\phi_{n}^{(0)}\right\}$ is linearly stable if and only if the perturbation $\left\{\epsilon_{n}(t)\right\}$ remains bounded in time, i.e. if and only if all eigenvalues $i \omega_{e}$ of the matrix in (12) are imaginary.

Note that writing the general solution of (111) as

$$
\epsilon_{n}(t)=\frac{1}{2}\left(a_{n}+b_{n}\right) e^{-i \omega_{e} t}+\frac{1}{2}\left(a_{n}^{*}-b_{n}^{*}\right) e^{i \omega_{e}^{*} t}
$$

leads to the following set of algebraic equations (e.g. [27]):

$$
\left\{\begin{array}{l}
\mathcal{L}_{0} b_{n}=\omega_{e} a_{n} \\
\mathcal{L}_{1} a_{n}=\omega_{e} b_{n}
\end{array}\right.
$$

with $\mathcal{L}_{0}$ and $\mathcal{L}_{1}$ defined above (13). Consequently, the condition of the linear stability of a solution $\left\{\phi_{n}^{(0)}\right\}$ is that all the eigenfrequencies $\omega_{e}$, obtained from (15), are real.

If $\omega_{e}$ is a real eigenfrequency of (15) and $\left(a_{n}, b_{n}\right)$ is the corresponding real solution, then $i \omega_{e}$ is an eigenvalue of (12) with eigenvector $\left(\left\{\alpha_{n} \equiv a_{n}\right\},\left\{\beta_{n} \equiv i b_{n}\right\}\right)$. Therefore with each real eigenfrequency $\omega_{e}>0$ one can associate a Krein signature $\kappa\left(\omega_{e}\right)$ (e.g. [35 27] and references therein):

$$
\kappa\left(\omega_{e}\right)=\operatorname{sign} \sum_{n} a_{n} b_{n} .
$$

For DGBs with frequencies inside the gap, eigenmodes with positive (negative) $\kappa$ correspond to excitations inside even (odd) waveguides in the AC limit $C \rightarrow 0$. Physically, the Krein signature is the sign of the Hamiltonian energy carried by the corresponding eigenmode (e.g. 35,36]).

\section{Construction of breather solutions}

The main idea for the numerical methods used to obtain breather solutions, based on the proof of their existence [1.37, is to perform numerical continuation of an exact breather solution, known for some particular parameter values, to other, arbitrary, values of these parameters. For stationary solutions (8), Eq. (11) with modulated coefficient $\lambda(2)$ reduces to a set of algebraic equations $(\gamma=1)^{2}$ :

$$
\begin{aligned}
& {\left[1+(-1)^{n} \delta^{2}-\omega_{b}\right] \phi_{n}-} \\
& \quad-C\left(\phi_{n-1}+\phi_{n+1}-2 \phi_{n}\right)+\left|\phi_{n}\right|^{2} \phi_{n}=0,
\end{aligned}
$$

and solutions can be found by Newton schemes (e.g. 38).

Usually breather solutions at $C=0$ (AC limit) are taken as initial solutions and continued to non-zero values of $C$. At $C=0$ one has an array of uncoupled waveguides. Hence, in the AC limit, for real solutions each amplitude $\phi_{n}$ should be taken from the set $\phi_{n} \in\left\{0, \pm A_{n}\right\}$, with:

$$
A_{n}=\sqrt{\omega_{b}-\lambda_{n}}
$$

Consequently, to each breather solution one can associate a corresponding coding sequence, where we use symbol

\footnotetext{
2 Note that the case of soft nonlinearity $\gamma=-1$ is also covered through the transformations $\phi_{n}^{\prime}=(-1)^{n} \phi_{n}, \omega_{b}^{\prime}=$ $2+4 C-\omega_{b}, \delta^{\prime 2}=-\delta^{2}$. Essentially, this reverses the roles of upper/lower bands and even/odd sites.
}

notation with up- and down- arrows for $+A_{n}$ and $-A_{n}$, respectively. To make the intrinsic structure of the solutions more clear, we use different sets of symbols for odd and even amplitudes (lower and upper band sub-fields): $\{\mathbb{O} \Uparrow \downarrow\}$ and $\{0 \uparrow \downarrow\}$, respectively.

Note, however, that these coding sequences are not unique for a given solution. Indeed, one can construct a DGB with some particular coding sequence and then continue it in frequency up to values above the upper gap boundary $\omega_{b}>\omega_{2}$. Above the gap this solution will have another coding sequence if continued to $C=0$, though the continuations in frequency and coupling can be done smoothly without any true bifurcations to other solutions. Similar effects were observed for second-harmonic penetration into a phonon band for breathers in a monoatomic Klein-Gordon lattice [39. In fact, one should distinguish two types of coding sequences: those corresponding to the $\mathrm{AC}$ limit inside and above the gap of the linear spectrum ('gap' and 'out-gap' coding sequences, respectively) ${ }^{3}$. We will use the superscripts $G$ or $O$ in coding sequences to indicate whether they are 'gap' or 'out-gap', respectively.

Restricting to solutions with spatial symmetry or antisymmetry with respect to the center, $N_{c}=\operatorname{Int}(N / 2)+1$, the coding sequences can be simplified by omitting the codes for sites $n=1,2 . . N_{c}-1$, and using subscripts $S$ or $A$ to mark whether a breather has symmetric or antisymmetric spatial configuration respectively. As follows from (18) and (2), in the case of hard nonlinearity $(\gamma>0$ in (10), the simplest symmetric DGBs are centered on a 'heavy' (odd) site with non-zero amplitude, while the simplest antisymmetric DGBs are centered on a 'light' (even) site with zero amplitude and with non-zero amplitudes of opposite signs on two neighboring 'heavy' sites.

Note that the structure of the linear spectrum, and in particular the gap boundaries $\omega_{1,2}$ (5), depends on the coupling $C$ (although the gap width $\Delta=2 \delta^{2}$ is independent on $C$ ). Thus, if one constructs a DGB with frequency $\omega_{b}$ inside the gap at some particular value of $C$ and tries to continue it versus $C$ at fixed frequency, it can reach one of the gap boundaries in the process of continuation. Consequently, a DGB can disappear (on the lower boundary) or transform into a DOGB (on the upper boundary) while continued in coupling. The same effect can occur for DOGBs, as the frequency may reach that of the linear wave corresponding to its tail. To avoid this problem we fix not the breather frequency, but the frequency detuning:

$$
\Delta \omega \equiv \omega_{b}-\omega_{o}(q, C)
$$

for the continuation in coupling. Here $\omega_{o}(q, C)$ is the frequency of the linear wave (44) with the same wave number $q$ as that of the breather tail, belonging to the upper branch of the spectrum. All stationary DGB solutions have tails with wave number $q=\pi / 2$, so that the frequency detuning (19) is equal to $\Delta \omega=\omega_{b}-\omega_{2}(C)<0$ for such breathers.

\footnotetext{
3 The existence of two types of coding sequences is connected to the existence of two types of AC limits. In general, the AC limit $\omega_{b} / C \rightarrow \infty$ can be reached either by taking $C=0$ or $\omega_{b} \rightarrow \infty$. These are not equivalent for modulated systems.
} 
Hence, the frequency detuning $\Delta \omega$ (19) is a more convenient parameter than the frequency $\omega_{b}$, and it can be considered as the only dynamical parameter of stationary DGBs and DOGBs. Note also that although Eq. (17) for stationary solutions has three parameters $\left(\omega_{b}, \delta^{2}, C\right)$, only two are independent, since varying the third parameter is equivalent to rescaling $\psi_{n}$. For instance, one can choose as independent parameters: $\delta^{2} / C$ - the 'modulation' parameter, and $\omega_{b} / C$ - the 'discreteness' parameter. In what follows we fix the 'gap' parameter $\delta^{2}$ and investigate the properties of stationary solutions while varying the frequency detuning $\Delta \omega$ (19) and the coupling $C$.

\section{Numerical results}

We here present the results of numerical continuation and stability analysis of DGB and DOGB solutions.

\subsection{Stability of gap breathers}

At frequencies inside the gap, $\omega_{1}<\omega_{b}<\omega_{2}$, only 'heavy' (odd) amplitudes $\phi_{n}$ can be non-zero in the AC limit (18). Thus, there are two basic configurations of DGB solutions with coding sequences $\{\Uparrow(0 \mathbb{O})\}_{S}^{G}$ and $\{0 \Uparrow(0 \mathbb{O})\}_{A}^{G}$ (codes in parenthesis are repeated), representing a symmetric DGB centered on a 'heavy' site with non-zero amplitude, and an antisymmetric DGB centered on a 'light' site with zero amplitude, respectively (Fig. 2). Since DGBs have exponentially decaying tails of wave number $q=\pi / 2$, neighboring even amplitudes, as well as neighboring odd amplitudes, have opposite signs. In addition, there is a phase shift of 'light' (even) amplitudes in the center (Fig. 2), known from gap solitons in the continuum limit (e.g. 29]).

In Fig. 3] we show the results of stability analysis of symmetric and antisymmetric DGBs for the continuation in coupling at three different frequency detunings (19): in the upper half of the gap, in the gap center, and in the lower half of the gap, respectively. The stability eigenvalues can be divided into two groups, corresponding to spatially localized and extended eigenvectors, respectively.

The 'extended' eigenvalues are independent on the breather spatial configuration and would be obtained also for the system without breather. They correspond to extended excitations of the upper and lower band sub-fields and form two bands $i \omega_{+}$and $i \omega_{-}$with positive and negative Krein signatures, respectively:

$$
\begin{aligned}
& i \omega_{+}=i\left(\omega_{o}(q)-\omega_{b}\right), \\
& i \omega_{-}=i\left(\omega_{b}-\omega_{a}(q)\right) .
\end{aligned}
$$

Here $\omega_{o}(q)$ and $\omega_{a}(q)$ are the frequencies of linear waves (3), (4) with wavenumbers $q$ (defined by the system size), corresponding to the upper and lower bands, respectively. In the limit of an infinite system the extended eigenvalues will have continuous spectrum. The boundaries of these bands are determined by the DGB frequency $\omega_{b}$ and the boundaries of the lower and upper bands of the linear spectrum $\omega_{1,2}, \omega_{u, d}$ (see Fig. 1). Defining the deviation $\alpha \equiv \Delta \omega+\delta^{2}$ of a DGB frequency from the gap center: $\omega_{b}=1+2 C+\alpha\left(-\delta^{2} \leq \alpha \leq \delta^{2}\right)$, yields

$$
\begin{aligned}
\delta^{2}-\alpha & \leq \omega_{+} \leq \sqrt{\delta^{4}+4 C^{2}}-\alpha, \\
\delta^{2}+\alpha & \leq \omega_{-} \leq \sqrt{\delta^{4}+4 C^{2}}+\alpha .
\end{aligned}
$$

Thus, having the same shape, the two bands of extended eigenvalues are shifted by $2 \alpha$ with respect to each other. While increasing the coupling constant $C$, these bands broaden and at a certain value $C_{0}$,

$$
C_{0}=\sqrt{\delta^{2}|\alpha|+\alpha^{2}}
$$

they overlap. (In the gap center $\alpha=0$, the two bands coincide as shown in Fig. 3(c),(d).) The overlapping of the bands will lead to collisions of extended eigenvalues with opposite Krein signatures with generation of instabilities (see Fig. 3). For larger systems more collisions will occur, as the bands become more 'dense', but the instabilities produced will become weaker and should completely disappear in the limit of an infinite system [40] ${ }^{4}$.

The localized eigenvalues play a more important role, as their collisions with each other or with extended eigenvalues can produce strong instabilities independent on the system size. The corresponding eigenvectors are localized around the breather when the eigenvalues lie outside the extended bands. There are two types of localized eigenvalues, bifurcating from the top of the extended band with $\kappa=+1(P 1, P 2, P 3, \ldots$ in Fig. 3(a)), and from the bottom of the band with $\kappa=-1(N 1, N 2)$, respectively. In addition, there is also the 'phase' mode with $i \omega_{e} \equiv 0$, whose eigenvector is the same as the breather solution, and associated with the phase rotation of the breather.

The eigenvector corresponding to the eigenvalue $N 1$ has symmetry opposite to the DGB. Colliding with its complex conjugate a real instability is produced, connected to the 'exchange of stability' between symmetric and antisymmetric DGBs ${ }^{5}$ [Peierls-Nabarro $(\mathrm{PN})$ mode, see below]. The other eigenvalue bifurcating from the negative Krein signature band, $N 2$, has an eigenvector with same symmetry as the breather. For DGB frequencies in the upper half of the gap, both $N 1$ and $N 2$ eigenvalues penetrate the positive Krein signature band, yielding oscillatory instability (Fig. 3(a), and N2 in (b)). In particular regimes the $N 2$ eigenmode can collide with its complex conjugate producing real instabilities, connected to transitions from 'discrete-like' to 'continuous-like' DGBs [17] (see Sec. 5.2)

The eigenvalues $P 1, P 2, P 3, \ldots$ bifurcate one by one when increasing the coupling, corresponding alternately to symmetric or antisymmetric eigenvectors. The number of these localized modes increases as, with increasing coupling, the central part of the DGB will occupy a larger number of sites. These eigenvalues can also produce oscillatory instabilities in the upper half of the gap, penetrating the extended band with opposite Krein signature

4 This is true generically for localized breathers, while for non-localized solutions (e.g. breathers with extended tails) such instabilities survive also in the infinite-size limit (e.g. 27]).

5 Here 'stability' only means w.r.t. the PN-mode, since Krein instabilities may exist also in this regime (see Fig. 31(a), (b)). 

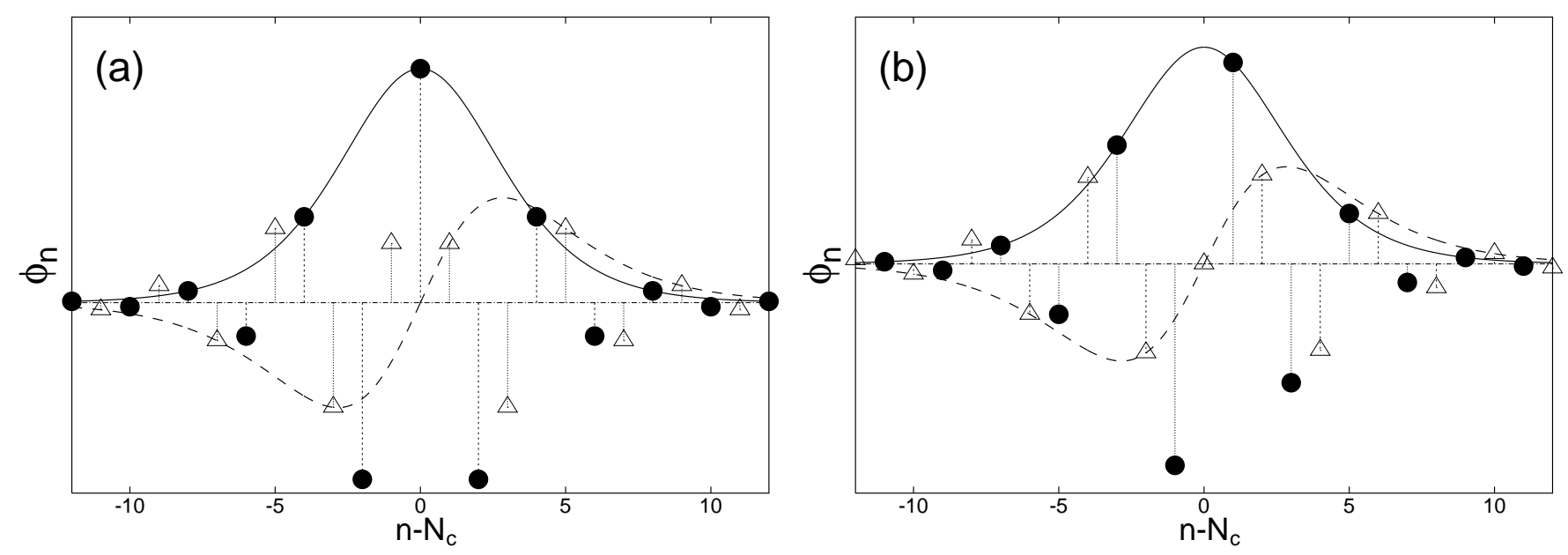

Fig. 2. Symmetric (a) and antisymmetric (b) DGBs at non-zero coupling. Circles (triangles) denote 'heavy' ('light') amplitudes.

(see Fig. 3(a),(b)). Unlike other eigenvalues, $P 1$ and $P 2$ (and for the antisymmetric DGB also $P 3$ ) can bifurcate from the extended band immediately when $C$ becomes non-zero. Analysis shows, that for each of these eigenvalues there is a critical value of the breather frequency detuning $\Delta \omega_{c r}$ (in the lower half of the gap for $P 1$ and $P 2$ ), specific for symmetric and antisymmetric DGBs, above which they stay outside the band (i.e., the eigenmodes are localized) at arbitrarily small non-zero coupling (see Appendix $\mathrm{A}$ for details). The deviation of these eigenvalues from the top of the extended band is proportional to $C^{2}$ at small values of $C$ (see Eqs.(32),(33)). By contrast, below $\Delta \omega_{c r}$ the eigenvalue is inside the band at small $C$.

Similar structure of extended and localized eigenvalues was reported for the diatomic KG model [17]. However, an important difference is that in the modulated DNLS model, DGBs with frequencies in the lower half of the gap do not possess any oscillatory instability associated with localized eigenvalues. Indeed, for frequencies in the lower half of the gap $(\alpha \leq 0$ in (21) $)$, the bottom of the extended band with $\kappa=-1$ is below the bottom of the $\kappa=+1$ band for all values of $C$ (see Fig. B](c)-(f)), so the $N 2$ eigenvalue does not penetrate the extended band. For similar reason the localized eigenvalues $P 1, P 2, P 3 \ldots$ do not penetrate the extended band, as the top of the $\kappa=+1$ band is above the top of the other band for all $C$. By contrast, in the diatomic KG model DGBs possess oscillatory instabilities associated with localized eigenmodes of $P$-type both in the upper and lower halves of the gap for larger values of the coupling, as the bands have different shapes [17].

The two types of oscillatory instabilities of DGB solutions were recently observed also in [20], where the stability of DGBs was analyzed for a continuation in frequency at a particular value of coupling within a similar model of a coupled waveguide array. The $P 1, P 2, P 3, \ldots$ instabilities can be associated with external resonances, as the frequencies of the corresponding localized modes are above the linear spectrum. By contrast, the N2 instability can be associated with internal resonances, as the frequency of this mode is inside the gap [20].

The real instability $N 1$ was also observed in [20] for the antisymmetric DGB solution, which was shown to be unstable at all frequencies inside the gap for the particular used value of coupling. However, we emphasize that in fact both symmetric and antisymmetric DGBs can possess this type of instability (see Fig. 3) at different values of coupling. This exchange of stability between symmetric and antisymmetric modes is very important for breather mobility issues 5.17. Generally, for the antisymmetric DGB at small $C>0$ there are two localized modes associated with antisymmetric and symmetric coupled oscillations in the two 'heavy' sites with non-zero codes. The former is the 'phase' mode with $i \omega_{e} \equiv 0$, while the latter is the $N 1$ mode which becomes unstable immediately when $C>0$ (see Fig. [3(b),(d),(f) and Appendix [A). Thus, antisymmetric DGBs always possess a real instability at small $C$, with a growth rate proportional to $C$ that can be obtained analytically (Appendix \$Table eigenmode $(N 1, A, G)$ ).

\subsection{Transition from DGB to DOGB solutions. Discrete- and continuous-like breathers.}

To study in details the transformation of DGBs into DOGBs, we perform the continuation in the frequency detuning (19) of the breather solutions, at fixed coupling. We focus our attention on symmetric breather configurations.

In Fig. 4 the profile of a symmetric breather is shown for the continuation in frequency detuning, at fixed coupling, from a value inside the gap to a value above the linear spectrum. At the upper gap boundary $(\Delta \omega=0)$ the DGB transforms into a DOGB with non-zero tails. The structure of this DOGB is qualitatively the same as that of a DGB (see Fig. 22(a)) but with non-zero asymptotes of the upper band sub-field ('light' amplitudes) $\left|\phi^{(u)}\right| \sim \sqrt{\Delta \omega}$, while the lower band sub-field ('heavy' amplitudes) still exponentially decays to zero. The wave number of the tails 

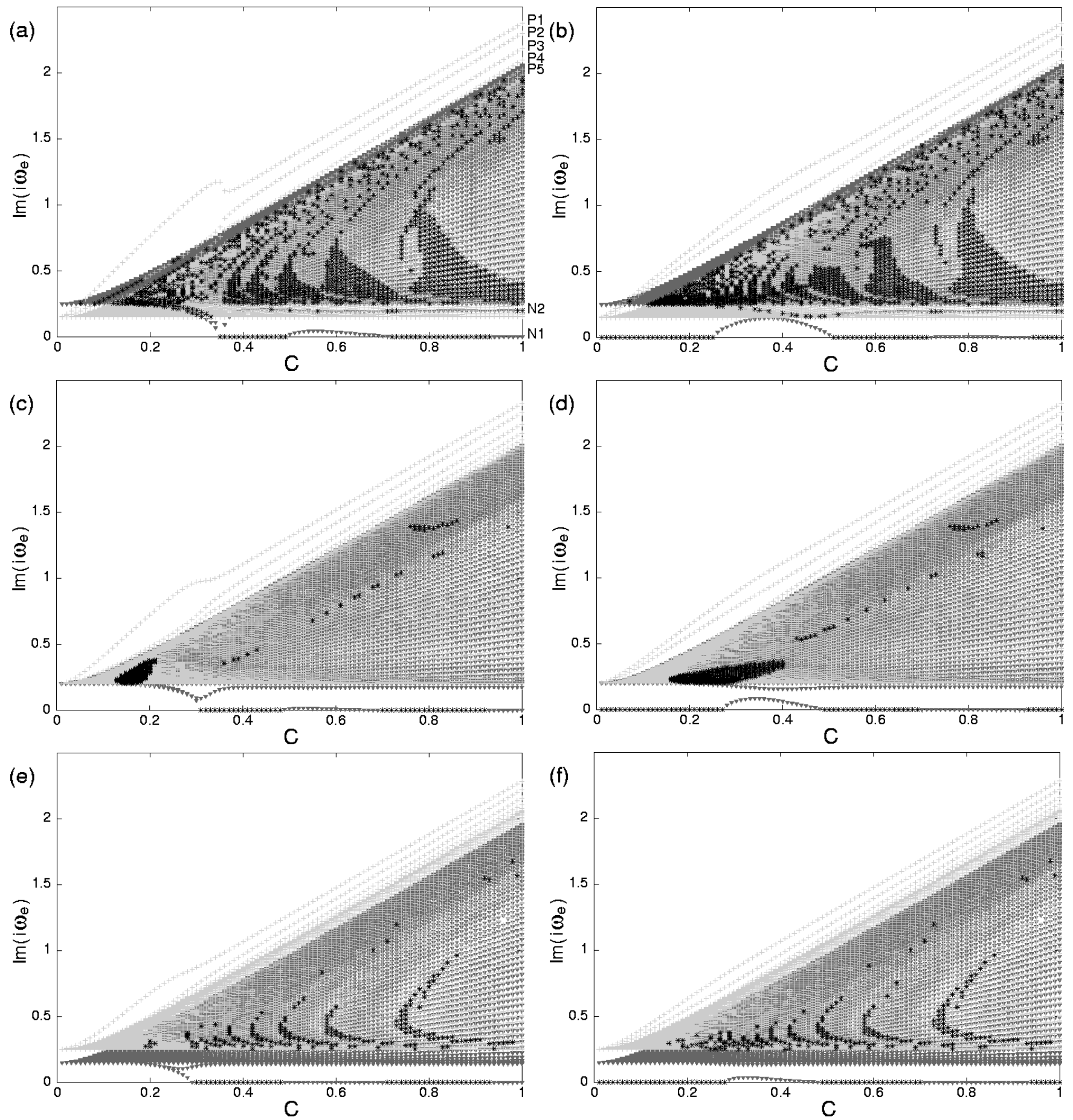

Fig. 3. Imaginary parts of eigenvalues $i \omega_{e}$ of (12) for symmetric (a),(c),(e) and antisymmetric (b), (d),(f) breathers at different values of the frequency detuning (19): (a),(b) $\Delta \omega=-0.75 \cdot \delta^{2}$ (upper half of the gap); (c),(d) $\Delta \omega=-\delta^{2}$ (middle of the gap); (e),(f) $\Delta \omega=-1.25 \cdot \delta^{2}$ (lower half of the gap). Light-grey crosses correspond to eigenvalues with positive Krein signature, dark-grey triangles to eigenvalues with negative Krein signature, and black stars to unstable eigenvalues. In all cases the system size $N=242$ and the gap parameter $\delta^{2}=0.2$. 


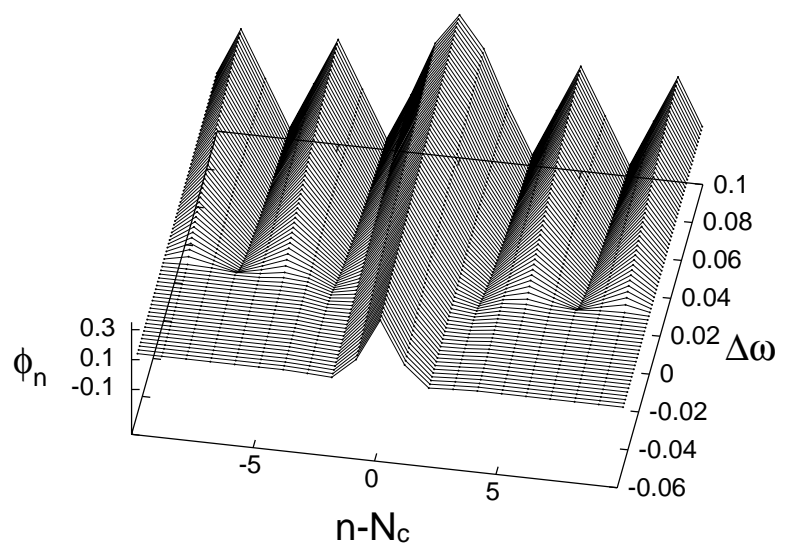

Fig. 4. Amplitudes of the central part of a breather for the continuation in frequency detuning $\Delta \omega$ from $\operatorname{DGB}\left(\Delta \omega=-0.6 \cdot \delta^{2}\right)$ to $\operatorname{DOGB}\left(\Delta \omega=\delta^{2}\right) . N=50, \delta^{2}=0.1$ and $C=0.02$.

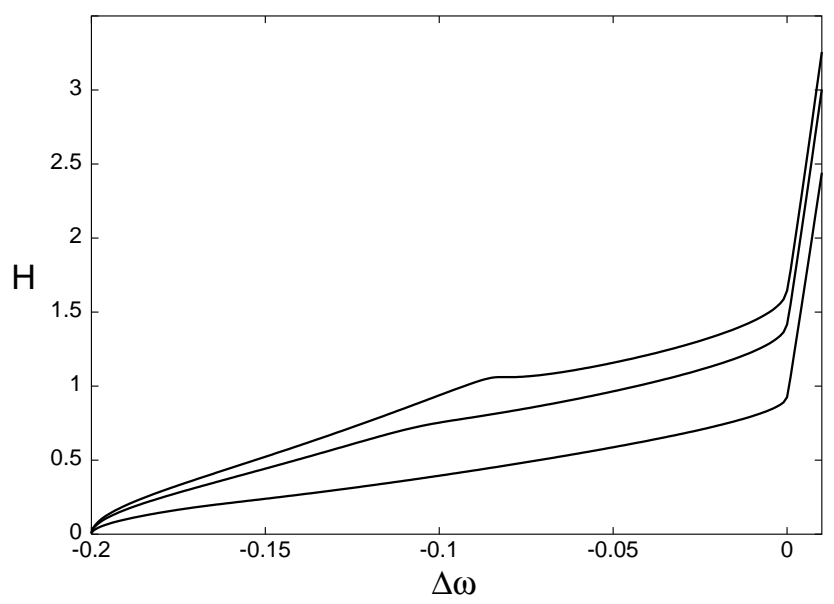

Fig. 5. The dependencies of the Hamiltonian energy $H$ (7) of a symmetric breather on its frequency detuning $\Delta \omega$ (19) for different values of the coupling (from top to bottom): $C=0.17$, $C=0.15$ and $C=0.1$. In all cases $\delta^{2}=0.1$ and $N=242$.

in this DOGB is $q=\pi / 2$ as for a DGB, and the corresponding coding sequence is $\{\Uparrow(\uparrow \mathbb{O} \downarrow \mathbb{O})\}_{S}^{O}$. In what follows we will call this configuration a 'normal' DOGB.

In Fig. 5 we show the dependencies of Hamiltonian $H$ (17) on frequency detuning $\Delta \omega$ (19), for continuation of symmetric breathers from $\Delta \omega=-2 \delta^{2}$ (bottom of the gap) to $\Delta \omega=0.1 \delta^{2}$ (inside the upper band) at different fixed values of the coupling. At the upper gap boundary $\Delta \omega=0$, a DGB $\{\Uparrow(0 \mathbb{O})\}_{S}^{G}$ transforms into a DOGB $\{\Uparrow(\uparrow \mathbb{O} \downarrow \mathbb{O})\}_{S}^{O}$, the energy of which rapidly grows with frequency due to the tails.

Increasing the coupling, the dependence $H(\Delta \omega)$ can become non-monotonous (e.g. $C=0.17$ in Fig. [5], which is connected to an additional transition from discrete- to continuous-like breather solution [17. Close to the AC limit $\left(C \ll 1\right.$ or $\left.\omega_{b} \gg \omega_{1}\right)$, the lower-band sub-field of the breather is well localized, and the two peaks of the upper-band sub-field (to the left and to the right of the breather center, see Fig. (2) are situated on the 'light' sites nearest to the breather center. We call this configuration 'discrete-like' breather. By contrast, close to the continuous limit the lower-band sub-field extends over many lattice sites, and the peaks of the upper band sub-field are located far from the breather center - 'continuous-like' breather. Consequently, the continuation of a breather from the anti-continuous to the continuous limit is always accompanied with an infinite number of tranformations, where each transformation corresponds to a 'jump' of upper band sub-field peaks from positions $n=N_{c} \pm n_{0}$ to positions $n=N_{c} \pm\left(n_{0}+2\right)$ with $n_{0}=1,3,5, \ldots$ for symmetric breathers and $n_{0}=2,4,6, \ldots$ for antisymmetric breathers.

In Fig. 6] different regimes of transitions from 'discretelike' breathers with peaks of the upper band sub-field at $n=N_{c} \pm 1$ (see insets in Fig. 6] a),(d)) to 'continuouslike' breathers with peaks of the upper band sub-field at $n=N_{c} \pm 3$ (insets in Fig. [6 (b), (e)) are demonstrated in the plane $(H, \Delta \omega)$ for continuation in frequency at different fixed values of coupling. For small enough $C$, a 'discretelike' breather smoothly transforms into a 'continuous-like' one while decreasing the frequency towards the lower gap boundary $\left(\Delta \omega \rightarrow-2 \delta^{2}\right)$. At a certain critical coupling $C_{1}$ the derivative $\partial H / \partial \omega_{b}$ turns to zero at the transition point (see Fig. [6(a)). Further increase of the coupling will lead to the appearence of a part of the $H(\Delta \omega)$ curve with a negative slope in the transition region (see Fig. 6b) b). As a consequence, discrete-like and continuous-like breathers will possess real instabilities of the Vakhitov-Kolokolov type [4] near the transition point. These instabilities are produced by the collisions of the $N 2$ localized eigenmode with its complex conjugate (see Sec. 5.1).

At the second critical value $C_{2}>C_{1}$ the slope of the 'unstable' part of the curve $H(\Delta \omega)$ will become vertical, and for $C>C_{2}$ it bends over and the dependence $H(\Delta \omega)$ becomes multi-valued in the transition region (Fig. 6(c) and lower curve in Fig. 6(d)). In this regime the transition between discrete-like and continuous-like breathers is possible only via an intermediate solution.

For large enough coupling, multi-breather solutions get involved in the transition from discrete-like to continuouslike breathers. In the upper right part of Fig. 6(d) the bifurcation loop for the breathers $\{\Uparrow 0 \Downarrow(\downarrow \mathbb{O} \uparrow \mathbb{O})\}_{S}^{O}$ (lower part of the loop) and $\{\Uparrow \downarrow \Downarrow(\downarrow \mathbb{O} \uparrow \mathbb{O})\}_{S}^{O}$ (upper part) is shown. Being constructed at frequencies above the linear spectrum, these DOGBs were continued in frequency down to the values inside the gap ${ }^{6}$, where they bifurcate with each other close to the bifurcation point of the continuous-like DGB with the intermediate breather. When the coupling reaches a third critical value $C_{3}$, these two bifurcation points coincide (Fig. [6(e)), and the four bifurcating solutions are identical in that point. For $C>C_{3}$ the bifurcation picture drastically changes. The curves for a continuous-like DGB $\{\Uparrow(0 \mathbb{O})\}_{S}^{G}$ and for a DOGB

\footnotetext{
${ }^{6}$ Note that these two solutions cannot be continued in frequency inside the gap at small values of coupling. Therefore, it is impossible to associate any 'gap' coding sequences to them.
} 

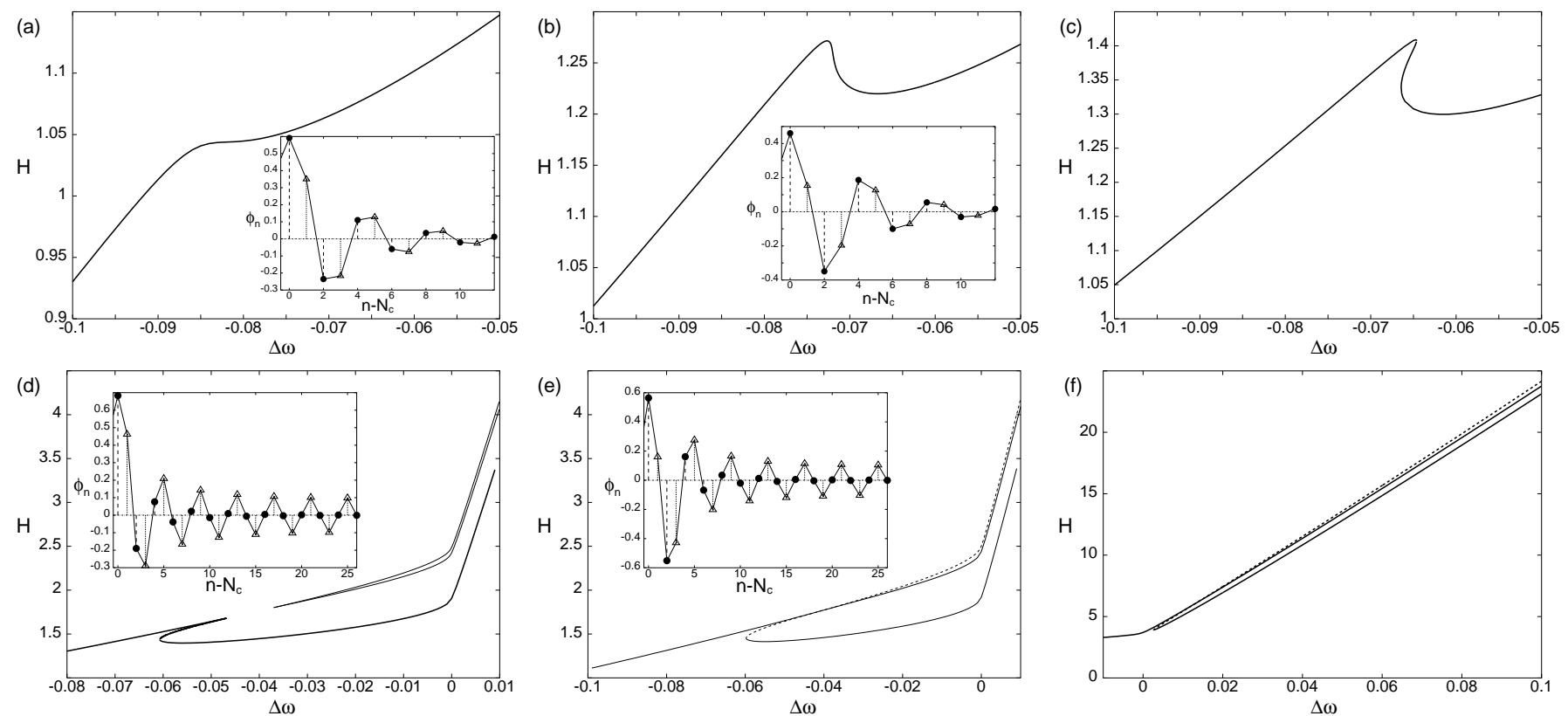

Fig. 6. Transitions from 'discrete-like' (larger $\Delta \omega$ ) to 'continuous-like' (smaller $\Delta \omega$ ) breathers. The dependencies of breather Hamiltonian energies $H$ (7) on frequency detunings $\Delta \omega$ (19) at different fixed values of the coupling: (a) $C=0.169$; (b) $C=0.18$; (c) $C=0.185$; (d) $C=0.191$; (e) $C=0.192$; (f) $C=0.28$. The insets in (a),(b),(d) and (e) show the structure of 'discrete-like' DGB $(C=0.169, \Delta \omega=-0.05)$, 'continuous-like' DGB $(C=0.18, \Delta \omega=-0.1)$, 'discrete-like' DOGB $(C=0.191, \Delta \omega=0.01$, lower curve) and 'continuous-like' DOGB $\left(C=0.192, \Delta \omega=0.01\right.$, middle curve), respectively. In all cases, $\delta^{2}=0.1$ and $N=242$.

$\{\Uparrow 0 \Downarrow(\downarrow \mathbb{O} \uparrow \mathbb{O})\}_{S}^{O}$ coincide, so that a single-breather solution DGB $\{\Uparrow(0 \mathbb{O})\}_{S}^{G}$ can be smoothly continued up to frequencies inside the linear spectrum (upper solid line in Fig. [6(e)). At the same time the curves for the intermediate breather, connecting the continuous-like and discretelike DGBs, and for the DOGB $\{\Uparrow \downarrow \downarrow(\downarrow \mathbb{O} \uparrow \mathbb{O})\}_{S}^{O}$ also coincide (dashed line in Fig. $6(\mathrm{e})$ ). Therefore, the discretelike DGB $\{\Uparrow(0 \mathbb{O})\}_{S}^{G}$ connected to the DOGB $\{\Uparrow(\uparrow \mathbb{O} \downarrow$ (O) $)\}_{S}^{O}$ now bifurcates with the DOGB $\{\Uparrow \downarrow \Downarrow(\downarrow \mathbb{O} \uparrow \mathbb{O})\}_{S}^{O}$ at frequencies inside the gap (lower solid line in Fig. 6(e)).

Further increase of $C$ will lead to the movement of the bifurcation point of the discrete-like DGB $\{\Uparrow(0 \mathbb{O})\}_{S}^{G}$ and the DOGB $\{\Uparrow \downarrow \Downarrow(\downarrow \mathbb{O} \uparrow \mathbb{O})\}_{S}^{O}$ towards the upper boundary of the gap. Finally, above the forth critical value of the coupling $C \geq C_{4}$, the discrete-like DOGB $\{\Uparrow(\uparrow \mathbb{O} \downarrow \mathbb{O})\}_{S}^{O}$, as well as the DOGB $\{\Uparrow \downarrow \downarrow(\downarrow \mathbb{O} \uparrow \mathbb{O})\}_{S}^{O}$ cannot be continued in frequency inside the gap, since they bifurcate with each other above the upper boundary of the gap $\Delta \omega=0$ (see Fig. G(f)). Consequently, for such large coupling only continuous-like DGBs $\{\Uparrow(0 \mathbb{O})\}_{S}^{G}$ can exist in the gap.

Similar transitions from discrete- to continuous-like breather solutions also occur for the continuation in coupling of DGBs and DOGBs at a fixed value of the frequency detuning $\Delta \omega$ (19). In the lower half of the gap $\left(-2 \delta^{2} \leq \Delta \omega \leq-\delta^{2}\right)$ the transitions are smooth. In the upper half of the gap the energy curve $H(C)$ bends over in a similar way as in Fig. 6(c), and the discrete-like breather transforms into a continuous-like one via an intermediate solution. Above the upper gap boundary $(\Delta \omega>0)$ the DOGBs $\{\Uparrow 0 \Downarrow(\downarrow \mathbb{O} \uparrow \mathbb{O})\}_{S}^{O}$ and $\{\Uparrow \downarrow \Downarrow(\downarrow \mathbb{O} \uparrow \mathbb{O})\}_{S}^{O}$ get involved in the transition between discrete- and continuous- like solutions (Fig. 17). In the lower left part of Fig. 7(a) the bifurcation loop for these two solutions is shown, while the upper right part shows the loop for transition from the continuous-like (upper solid line) into the discrete-like (lower solid line) DOGBs $\{\Uparrow(\uparrow \mathbb{O} \downarrow \mathbb{O})\}_{S}^{O}$ via the intermediate out-gap breather (dashed line). At a certain critical $\Delta \omega$ the bifurcation point of the DOGBs $\{\Uparrow 0 \Downarrow(\downarrow$ $\mathbb{( O )}(\mathbb{O})\}_{S}^{O}$ and $\{\Uparrow \downarrow \Downarrow(\downarrow \mathbb{O} \uparrow \mathbb{O})\}_{S}^{O}$ coincide with that of the continuous-like and intermediate out-gap breathers. At higher frequencies the continuous-like DOGB can be continued smoothly in coupling down to $C=0$, with the coding sequence $\{\Uparrow 0 \Downarrow(\downarrow \mathbb{O} \uparrow \mathbb{O})\}_{S}^{O}$ (upper solid line in Fig. [7 (b)), while the discrete-like DOGB $\{\Uparrow(\uparrow \mathbb{O} \downarrow \mathbb{O})\}_{S}^{O}$ bifurcates with the DOGB $\{\Uparrow \downarrow \Downarrow(\downarrow \mathbb{O} \uparrow \mathbb{O})\}_{S}^{O}$ (lower solid and dashed lines in Fig. (7) (b), respectively). An analogous scenario will then be repeated at higher frequencies, for the transition from a solution with peaks of the upper band sub-field at $n=N_{c} \pm 3$ to one with upper band sub-field peaks at $n=N_{c} \pm 5$. In Fig. Z(b) one can see a bending of the $H(C)$ curve for the continuous-like solution at $C \approx 0.68$, associated with this subsequent transition.

A similar switching of the bifurcation scenario with change of coupling was also observed for 'phantom' breathers associated with second-harmonic resonances in monoatomic KG chains [39].

\subsection{Bifurcations of DOGBs with different tails}

As described above, gap breathers $\{\Uparrow(0 \mathbb{O})\}_{S}^{G}$ gain nondecaying tails with wave number $q=\pi / 2$ when continued 

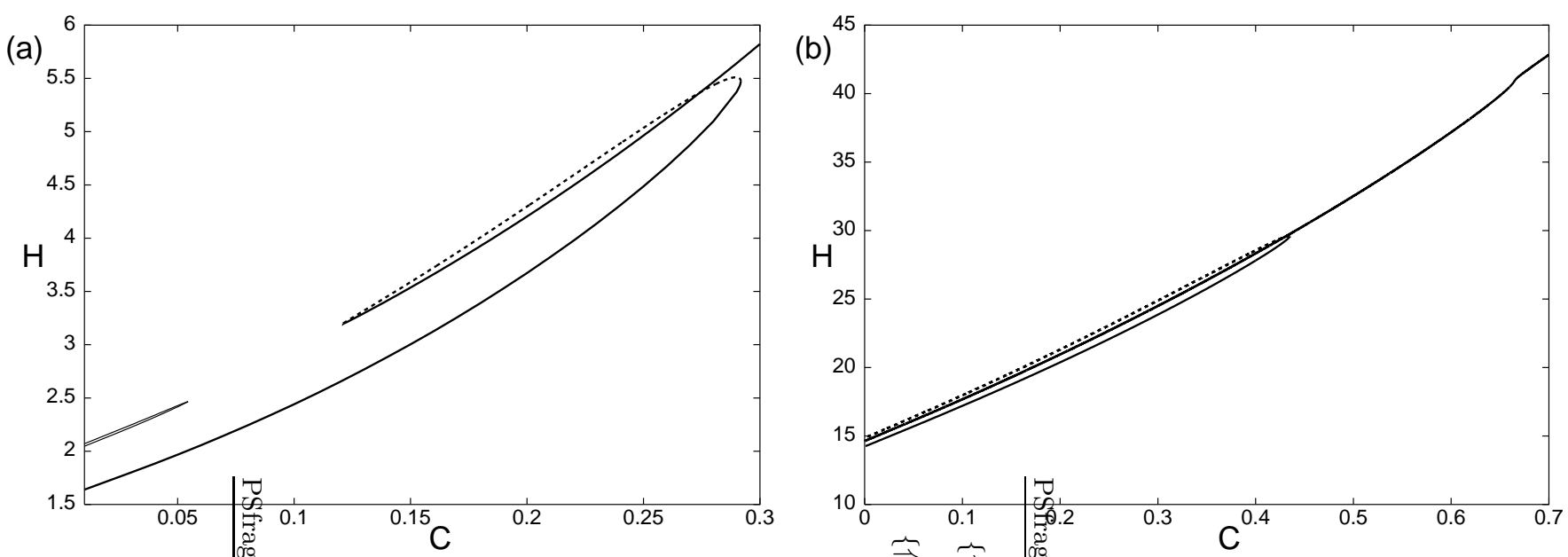

Fig. 7. Transitions from 'discrete-like' to 'continuous-like' out-gap breathers (see text for explanations). The dependencies of breather Hamiltonian energies $H$ (7) on the coupling $C$ at different fixed values of the frequency detuning $\Delta \omega$ : (a) $\Delta \omega=0.1 \cdot \delta^{2}$ and (b) $\Delta \omega=\delta^{2}$. The values of the system parameters are the same as in Fig. [6]
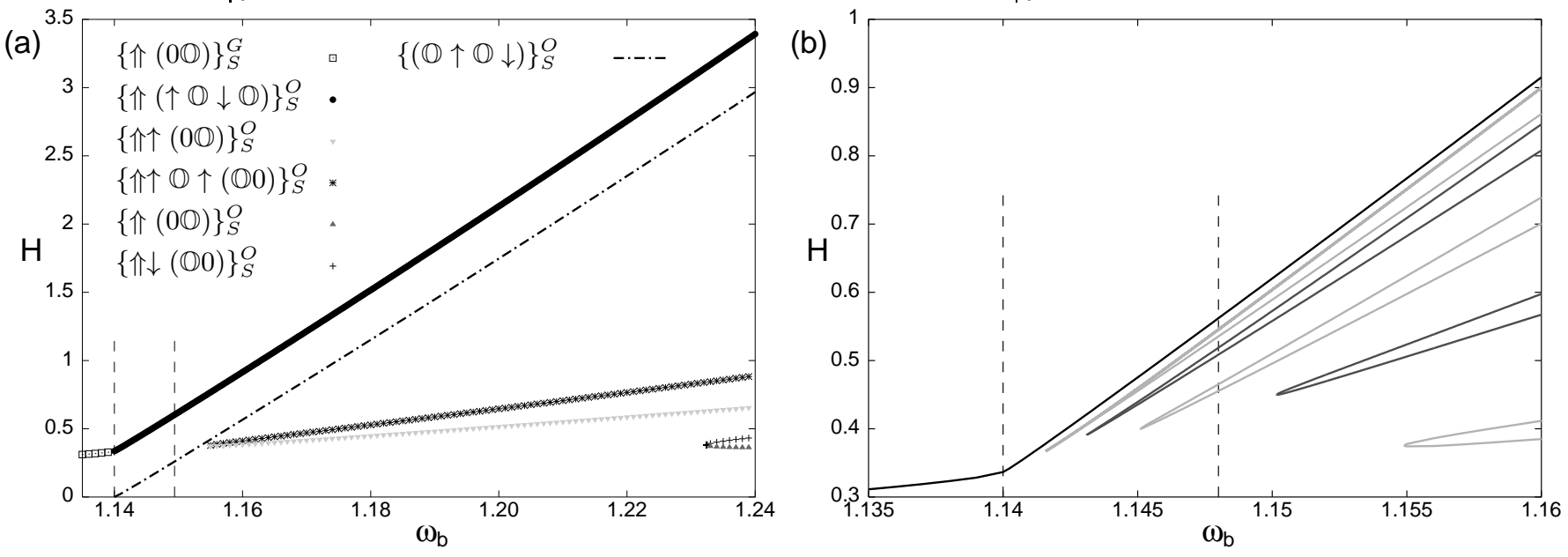

Fig. 8. Hamiltonian $H$ (7) vs. frequency $\omega_{b}$ for different solutions. Vertical lines show the boundaries of the upper branch of the linear spectrum $\omega_{2}=1.14, \omega_{u} \approx 1.148$. Curves in $(\mathrm{b})$ show, from top to bottom: continuation DGB $\{\Uparrow(0 \mathbb{O})\}_{S}^{G} \rightarrow \mathrm{DOGB}$ $\{\Uparrow(\uparrow \mathbb{O} \downarrow \mathbb{O})\}_{S}^{O}$, bifurcation loops for DOGBs with 1,2,4,8 and 12 'defects' (see text for explanation). $\delta^{2}=0.1, C=0.02, N=50$.

into 'normal' DOGBs above the upper gap boundary, and their energies rapidly grow. However, there exist also localized breathers with frequencies above the linear spectrum - 'on-top' breathers (DOTB), with exponentially decaying tails of wave number $q=\pi$ (for stationary solutions). The simplest example is the single-site DOTB with coding sequence $\{\Uparrow(0 \mathbb{O})\}_{S}^{O}$. Attempting a continuation of this solution in frequency down to the linear spectrum, it bifurcates with another on-top breather $\{\Uparrow \downarrow(\mathbb{O} 0)\}_{S}^{O}$ rather far from the 'top' frequency $\omega_{u}$ of linear waves (Fig. 8] (a), lowest curves). Another DOTB $\{\Uparrow \uparrow(\mathbb{O} 0)\}_{S}^{O}$ (three-site breather) can be continued much further down in frequency, bifurcating with the DOTB $\{\Uparrow \uparrow \mathbb{O} \uparrow(\mathbb{O} 0)\}_{S}^{O}$ close to the point $\omega=\omega_{u}$ (Fig. 8 (a), middle curves). The slope of the curve $H\left(\omega_{b}\right)$ for the DOTB $\{\Uparrow \uparrow(\mathbb{O} 0)\}_{S}^{O}$ is approximately the same as for the DGB $\{\Uparrow(0 \mathbb{O})\}_{S}^{G}$. In that sense this ontop breather, rather than the DOTB $\{\Uparrow(0 \mathbb{O})\}_{S}^{O}$, can be considered as the 'on-top counterpart' of the gap breather (however, this is true only for small coupling, see below).

Besides the 'normal' DOGBs, another type of out-gap breather with tail wave number $q=\pi / 2$ exists above the gap - the DOGB $\{(\mathbb{O} \uparrow \mathbb{O} \downarrow)\}_{S}^{O}$ (and its antisymmetric counterpart $\left.\{0(\mathbb{O} \uparrow \mathbb{O} \downarrow)\}_{A}^{O}\right)$. The dependence $H\left(\omega_{b}\right)$ for the continuation in frequency of this DOGB at fixed coupling $C=0.02$ is shown in Fig. 8 (a) (dash-dotted line). Originating from the linear wave with $q=\pi / 2$ at the upper gap boundary, it represents a kink-like excitation of 'light' sites ('phase domain wall') accompanied by localized excitation of 'heavy' sites at non-zero coupling, and corresponds to the kink-like out-gap soliton in the continuous limit (e.g. 7 29]). However, above a critical coupling $C_{c r} \approx 0.188$ the DOGB $\{(\mathbb{O} \uparrow \mathbb{O} \downarrow)\}_{S}^{O}$ does not exist at $\Delta \omega \rightarrow 0$, since being continued in frequency down towards the upper gap boundary at fixed coupling $C>C_{c r}$, it bi- 
furcates with the solution $\{\Downarrow \downarrow(\mathbb{O} \downarrow \mathbb{O} \uparrow)\}_{S}^{O}$ at $\Delta \omega>0$. Instead, at $C>C_{c r}$ the solution $\{\Downarrow 0(\mathbb{O} \downarrow \mathbb{O} \uparrow)\}_{S}^{O}$ can be continued down to the upper gap boundary, corresponding to the kink-like soliton in this regime. This is similar to the switching of the coding sequence for the continuous-like 'normal' DOGB from $\{\Uparrow(\uparrow \mathbb{O} \downarrow \mathbb{O})\}_{S}^{O}$ to $\{\Uparrow 0 \Downarrow(\downarrow \mathbb{O} \uparrow \mathbb{O})\}_{S}^{O}$ discussed in Sec. 5.2

Apart from DOGBs with tail wave numbers $q=\pi / 2$ and DOTBs with $q=\pi$, one can also construct DOGBs with any other tail structure, if the chosen wave number is allowed for the particular system size. As the coding sequences become complicated, we introduce a simplified notation. Restricting to symmetric breather configurations we omit the subscripts $S$, and likewise the superscripts $O$ will be omitted as all DOGBs are constructed at frequencies above the gap. For DOGBs with frequencies inside the linear spectrum the upper band sub-field ('light' amplitudes) should have non-zero tails because of resonances with linear waves. Therefore, the codes for 'light' amplitudes should be, generally, non-zero. Restricting to DOGBs which are 'single-site' in the lower band sub-field, all codes for 'heavy' sites are zero except for the central excited site, and the codes for 'heavy' amplitudes can be omitted. For example, the simplified coding sequence for a $\operatorname{DOGB}\{\Uparrow(0 \mathbb{O})\}_{S}^{O}$ is $[(0)]$, and for DOGB $\{\Uparrow(\uparrow \mathbb{O} \downarrow \mathbb{O})\}_{S}^{O}$ is $[(\uparrow \downarrow)]$ (we use different types of brackets to distinguish normal and simplified coding sequences).

To understand the total bifurcation picture of DOGBs with different tails, we construct new solutions by inserting 'defects' into the tail structure of a DOGB $[(\uparrow \downarrow)]$. Considering this sequence as the 'right' one, we replace the 'right' code at some particular place $k$ with a 'wrong' one together with introducing an additional phase shift, i.e. a change of the signs of all the subsequent codes (i.e. we insert a defect into the 'right' coding sequence to obtain a standing wave with wave number smaller than $q=\pi / 2$ ). For instance, denoting the number of codes in the simplified coding sequence as $N_{L}$ (i.e. the number of 'light' sites to the right of the breather center, e.g. $N_{L}=13$ if $N=50$ ), the solution with one 'defect' at the position $k$ (in the simplified coding sequence, corresponding to site $\left.n=N_{c}+2 k-1\right)$ has the simplified coding sequence: $\left[\left(r_{i}\right)_{i=1,2, \ldots, k-1}, w_{k},\left(-r_{i}\right)_{i=k+1, k+2, \ldots, N_{L}}\right]$. Here $r_{i}$ is the 'right' code at the $i$-th position, and $w_{k}$ is a 'wrong' code ( $w_{k}$ is ' 0 ' or ' $\downarrow$ ' if $r_{k}$ is ' $\uparrow$ ', $w_{k}$ is ' 0 ' or ' $\uparrow$ ' if $r_{k}$ is ' $\downarrow$ '). A DOGB $[0(\uparrow \downarrow)]$ is an example of a solution with a defect at the first position, while a DOGB $[\uparrow \downarrow \uparrow \uparrow(\downarrow \uparrow)]$ has a defect at the 4 th postition. Similarly DOGBs with more defects can be constructed. A single-site DOTB [(0)] can be considered as a solution with $N_{L}$ 'defects'.

We find, that being continued down in frequency towards the linear spectrum, each solution with 'defects' bifurcates with a solution having the same number of 'defects', with coding sequences differing only at the 'defects'

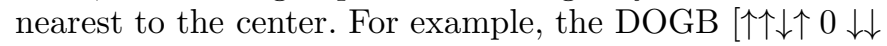
$(\uparrow \downarrow)]$ with three 'defects' at 2nd, 5th and 7th positions bifurcates with the DOGB $[\uparrow 0 \downarrow \uparrow 0 \downarrow \downarrow(\uparrow \downarrow)]$, with a different type of 'defect' at the 2 nd position. The resulting bifurcation picture is shown in Fig. 8(b), where bifurca-

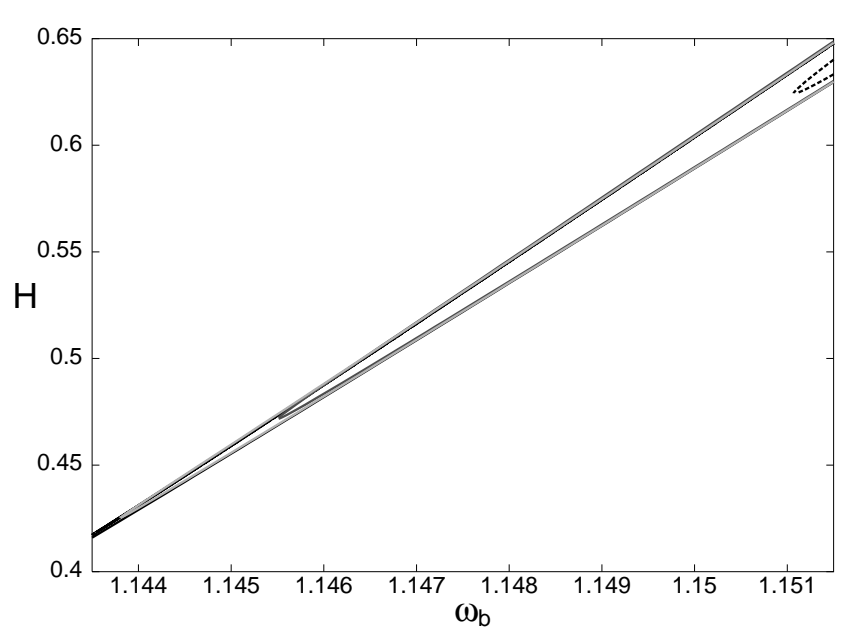

Fig. 9. The intrinsic structure of the bifurcation loop for the DOGB with one 'defect' in Fig. 8(b). Dashed black, solid darkgrey, solid light-grey and solid black lines represent solutions with one 'defect' at 1st, $2 \mathrm{nd}$, 3rd and 9 th positions respectively.

tion loops for solutions with 1,2,4,8 and 12 'defects' are plotted in different greyscales, while the solid black curve corresponds to the transition DGB $\{\Uparrow(0 \mathbb{O})\}_{S}^{G} \rightarrow$ DOGB $[(\uparrow \downarrow)]$. Note that in Fig. $8 N_{L}=13$, and the bifurcation loop for solutions with 12 'defects' in Fig. [8(b) is the same as that for the DOTBs $[\uparrow(0)]$ and $[\uparrow \uparrow(0)]$ in Fig. $[8$ (a).

Each bifurcation loop corresponding to a particular number of 'defects' has a complex intrinsic structure, as these 'defects' can be at different positions. For each number of defects one can find some 'optimal' configuration, so that the solution with 'defects' placed on these 'optimal' positions can be continued further down in frequency than all other solutions with the same number of 'defects'. The loops shown in Fig. 8(b) represent such optimal configurations. In Fig. 9 the intrinsic structure of the bifurcation loop for solutions with one 'defect' at 1st, 2nd, 3rd and 9th ('optimal') positions, respectively, is shown.

Increasing the coupling, the bifurcation picture remains qualitatively the same. However, the slope of the curve $H\left(\omega_{b}\right)$ for the DGB $\{\Uparrow(0 \mathbb{O})\}_{S}^{G}$ changes, so that for larger coupling, when the DGB has 'continuous-like' structure with upper-band sub-field peaks at $n=N_{c} \pm 3$, its 'ontop' counterpart (with approximately the same slope for $\left.H\left(\omega_{b}\right)\right)$ is the DOTB $\{\Uparrow \uparrow \mathbb{O} \downarrow(\mathbb{O} 0)\}_{S}^{O}$. Similar changes of the DGB's 'on-top' counterpart are expected for a further increase of coupling, as the peaks of the upper-band sub-field will move further away from the breather center.

The bifurcation scheme for out-gap breathers in Fig. 8 (b) is qualitatively similar to that for phonobreathers with different tails resulting from second-harmonic resonances in monoatomic KG chains 39 . However, in the modulated DNLS model out-gap breather tails apparently always have non-zero asymptotes, and thus we found no analogue to 'phantom' breathers [39] with vanishing tails. 

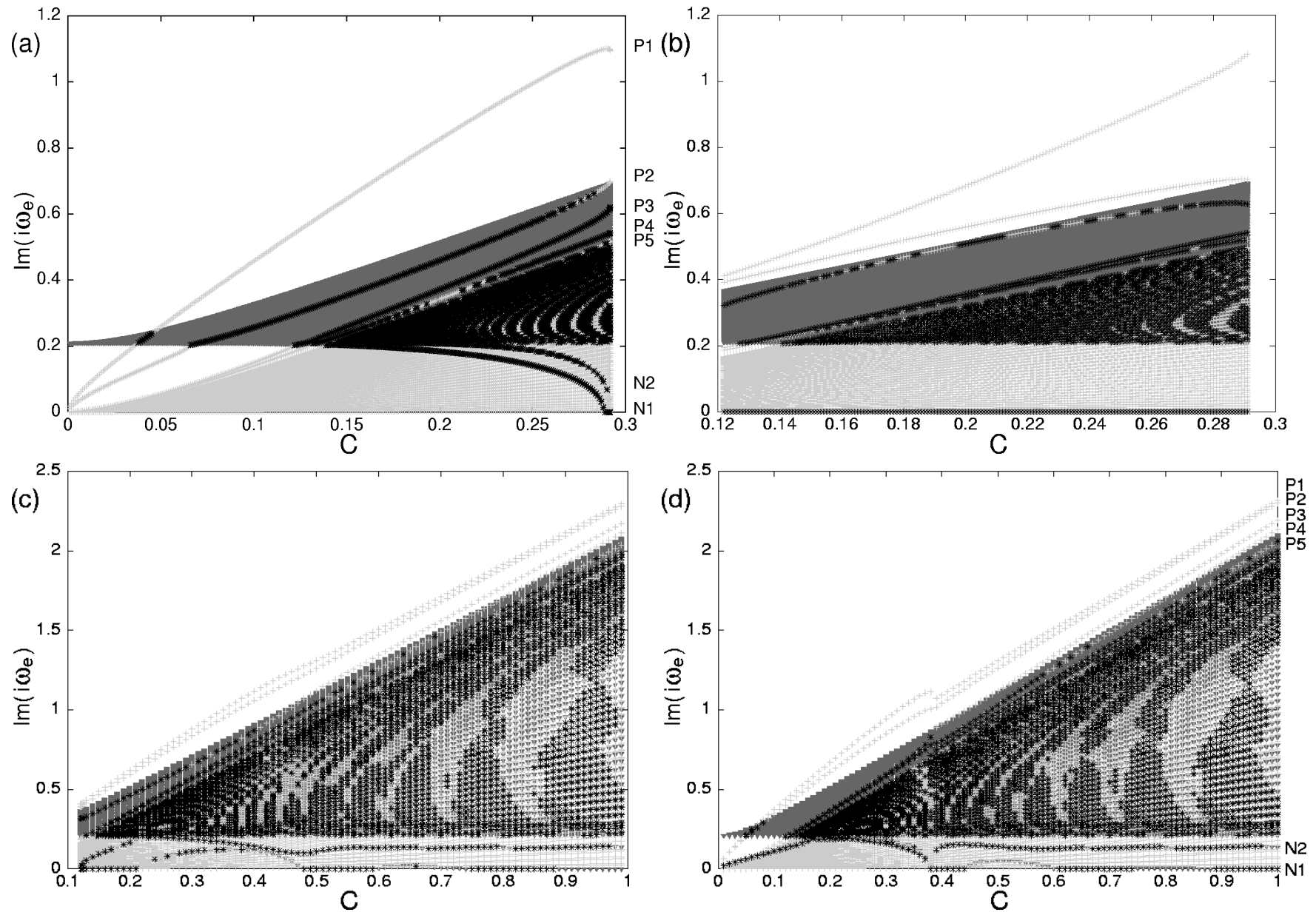

Fig. 10. Imaginary parts of eigenvalues $i \omega_{e}$ of (12) for symmetric discrete-like (a), intermediate (b), continuous-like (c) and antisymmetric (d) 'normal' DOGBs at frequency deviation (19) $\Delta \omega=0.1 \cdot \delta^{2}$. Light-grey crosses (dark-grey triangles) correspond to eigenvalues with $\kappa=+1(\kappa=-1)$, and black stars to unstable eigenvalues. (a)-(c) correspond to upper right loop in Fig. [7(a).

\subsection{Stability of out-gap breathers}

We analyze the stability of DOGBs by continuation in coupling at fixed frequency detuning (19), focusing on the three pairs of most important solutions: 'normal' DOGBs $\{\Uparrow(\uparrow \mathbb{O} \downarrow \mathbb{O})\}_{S}^{O},\{0 \Uparrow(\uparrow \mathbb{O} \downarrow \mathbb{O})\}_{A}^{O}$, being the smooth continuation of symmetric and antisymmetric DGBs in frequency above the gap for small coupling; DOTBs $\{\Uparrow$ $(0 \mathbb{O})\}_{S}^{O},\{0 \Uparrow(0 \mathbb{O})\}_{A}^{O}$, being the fundamental 'on-top' breathers; and DOTBs $\{\Uparrow \uparrow(\mathbb{O} 0)\}_{S}^{O},\{0 \Uparrow \uparrow(\mathbb{O} 0)\}_{A}^{O}$, being the 'on-top counterparts' of DGBs for small coupling. We fix the system size $N=242$ and gap parameter $\delta^{2}=0.1$.

\subsection{1 'Normal' DOGBs}

In Fig. 10 we show stability results for symmetric discretelike, intermediate, continuous-like, and antisymmetric outgap breathers at frequency detuning $\Delta \omega=0.1 \cdot \delta^{2}$. (Note, that in 'normal' DOGBs the tail wave-number is $q=\pi / 2$, so according to (19) $\Delta \omega=\omega_{b}-\omega_{o}(\pi / 2) \equiv \omega_{b}-\omega_{2}$.) As for DGBs (Sec. 5.11), there are two bands of extended eigen- values with opposite Krein signatures:

$$
\begin{aligned}
i \omega_{\mp} & =\left\{\frac{\left(\Delta \omega+2 \delta^{2}\right)^{2}+8 C^{2} \cos ^{2}(q)}{2} \pm\right. \\
& \left. \pm \frac{1}{2} \sqrt{\left(\Delta \omega+2 \delta^{2}\right)^{4}+16 C^{2} \cos ^{2}(q)\left(4 \delta^{4}-\Delta \omega^{2}\right)}\right\}^{\frac{1}{2}}
\end{aligned}
$$

with all wavenumbers $q$ allowed for the particular system size. But now the band $\left\{i \omega_{+}\right\}$with positive Krein signature always has its lower boundary at zero. Moreover, as for non-localized solutions Krein instabilities corresponding to extended eigenmodes survive also for infinite systems (e.g. [27]), overlapping of the extended bands is more essential for extended DOGBs than for localized DGBs.

Increasing the coupling, several localized modes bifurcate from the top of the band with $\kappa=+1(P 1-P 5$ in Fig. 10] (a),(d)) and from the bottom of the band with $\kappa=-1$ (N1 and N2 in Fig. 10 (a),(d)). Penetrating the bands with opposite Krein signature, these modes produce oscillatory instabilities. The $N 1$ mode also causes a real instability with an eigenvector of opposite symmetry to the DOGB, colliding with its complex conjugate at zero (PN 


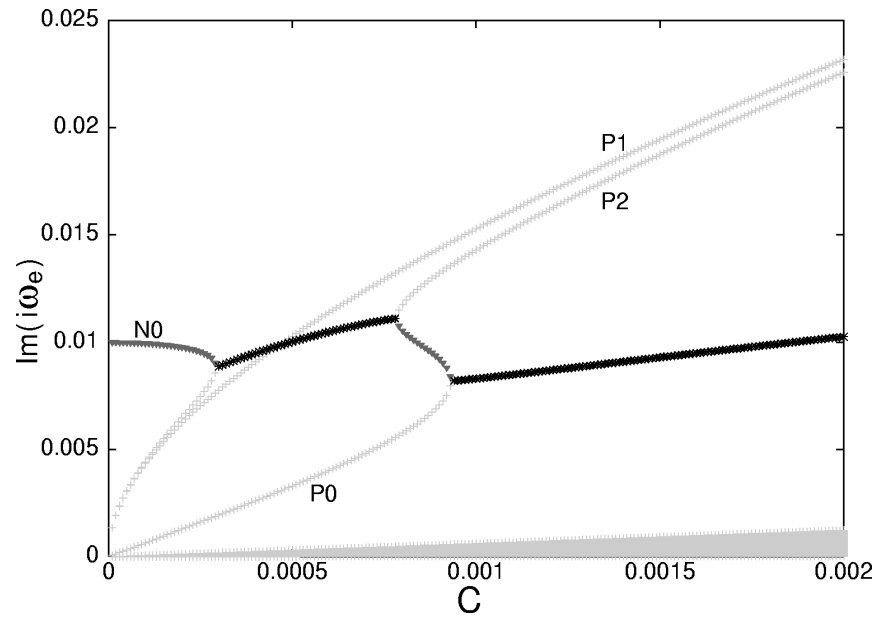

Fig. 11. Blow-up of a regime close to the origin of Fig. [10(d).

instability yielding 'exchange of stability' ${ }^{7}$, between symmetric and antisymmetric DOGBs, cf. Sec. 5.1). Similarly, the $N 2$ mode causes a real instability with an eigenvector of the same symmetry as the DOGB, in the regime of transition from discrete-like to continuous-like solutions.

However, the behaviour of the localized modes at small coupling is different for gap and out-gap breathers. For symmetric DGBs and DOGBs, in the limit of small $C$ only two modes can stay localized: $P 1$ and $P 2$, associated with symmetric and antisymmetric oscillations on the sites closest to the breather center. In the case of DOGBs, in contrast to DGBs, there is no threshold $\Delta \omega_{c r}$, and these two eigenvalues bifurcate from the extended band immediately when $C$ becomes non-zero, for all DOGB frequencies (see Appendix A Table 1). The differences between each of the $P 1$ and $P 2$ eigenvalues and the top boundary of the extended band are in this case proportional to $\sqrt{C}$ at small values of $C$ (see Fig. 10 and Table 1] compare with $C^{2}$-dependence for DGBs as discussed in Sec. [5.1)

For antisymmetric 'normal' DOGB $\{0 \Uparrow(\uparrow \mathbb{O} \downarrow \mathbb{O})\}_{A}^{O}$ an additional mode $N 0$ appears (see Fig. [11), localized on the 'central' site $N_{c}$ which is the only 'light' site with code ' 0 '. Note, that in an antisymmetric DGB $\{0 \Uparrow(0 \mathbb{O})\}_{A}^{G}$ all 'light' sites have zero codes, therefore there is no such localized mode for breather frequencies inside the gap.

Another important difference between antisymmetric 'normal' DOGBs and DGBs is, that while the latter always have a real instability at small coupling (see Sec. 5.11), the former are always stable in a small interval of $C$ : $\left[0, C_{0}\right]$ (see Fig. 111). A localized mode $P 0$, with eigenvector of opposite symmetry to the DOGB, now bifurcates from the upper boundary of the positive Krein signature band, similarly to the $P 1$ and $P 2$ modes. The eigenfrequency is proportional to $C$ for small $C$ (see Table 1 in Appendix eigenmode $(P 0, A, O))$, and the eigenvector for $C \rightarrow 0$ involves oscillations not only in the 'excited' 'heavy' sites, but also in the subsequent 'light' sites. At a certain nonzero value of $C$ it collides with the $N 0$ mode producing

\footnotetext{
${ }^{7}$ Krein instabilities generally also exist here, see Fig. 10]
}

an oscillatory instability, associated with a resonance between oscillations in the central 'light' site and symmetric coupled oscillations in the two neighboring 'heavy' sites.

The $N 1$ localized mode, which is responsible for the 'exchange of stability' between antisymmetric and symmetric 'normal' DOGBs, now appears only at large coupling, in the continuous-like regime.

\subsection{2 'On-top' DOGBs}

As indicated in Sec. [5.3] 'on-top' breathers cannot be continued in frequency down to the linear spectrum ${ }^{8}$. Analogously they cannot be continued in coupling up to arbitrary values at fixed frequency detuning $\Delta \omega$, since they bifurcate with other DOTBs in the same way as when continued in frequency at fixed $C$ (see Fig. 88(a)). By contrast, the DOTBs can be smoothly continued by simultaneously increasing the frequency detuning and the coupling at fixed value of the ratio $\Delta \omega / C$. This corresponds to the continuation towards the 'non-modulated' limit $\delta^{2} / C \rightarrow 0$ (see Eq. (17)), i.e. towards usual 'on-top' breathers in homogeneous media. Only for $\delta^{2} / C=0$ the smooth continuation in coupling at fixed frequency detuning can be performed; at any non-zero $\delta^{2} / C$ the exact limit $\Delta \omega / C \rightarrow 0$ cannot be reached because of the bifurcations.

In Fig. 12. results of continuation in coupling of DOTBs $\{\Uparrow(0 \mathbb{O})\}_{S}^{O},\{0 \Uparrow(0 \mathbb{O})\}_{A}^{O},\{\Uparrow \uparrow(\mathbb{O} 0)\}_{S}^{O}$ and $\{0 \Uparrow \uparrow(\mathbb{O} 0)\}_{A}^{O}$ at fixed frequency detuning are shown. Each DOTB is continued from $C=0$ up to the bifurcation point, where it bifurcates with DOTBs $\{\Uparrow \downarrow(\mathbb{O} 0)\}_{S}^{O},\{0 \Uparrow \downarrow(\mathbb{O} 0)\}_{A}^{O}$, $\{\Uparrow \uparrow \mathbb{O} \uparrow(\mathbb{O} 0)\}_{S}^{O}$ and $\{0 \Uparrow \uparrow \mathbb{O} \uparrow(\mathbb{O} 0)\}_{A}^{O}$, respectively. For all DOTBs the extended eigenvalues have the same (negative) Krein signature. Still they can be divided into two bands ('upper' and 'lower'):

$$
\begin{aligned}
i \omega_{u p} & =\omega_{b}-\omega_{o}(q), \\
i \omega_{l o} & =\omega_{b}-\omega_{a}(q),
\end{aligned}
$$

where $\omega_{o, a}(q)$ are frequencies from the upper and lower bands of the linear spectrum (4).

Close to the bifurcation point, all the described DOTBs possess two real instabilities with symmetric and antisymmetric eigenvectors, respectively. These instabilities are similar to those produced by the $N 1$ and $N 2$ modes in the case of DGBs and 'normal' DOGBs in the region of transition from discrete- to continuous-like solutions.

Like 'normal' antisymmetric DOGBs, antisymmetric DOTBs have no instabilities at small coupling, so for each pair of symmetric and antisymmetric DOTBs there is an interval $\left[0, C_{0}\right]$ of simultaneous stability. The localized mode associated with symmetric coupled oscillations in the two 'excited' 'heavy' sites (similar to the $P 0$ mode for a 'normal' antisymmetric DOGB) bifurcates from zero and, having $\kappa>0$, collides with another localized mode associated with oscillations in the 'central' 'light' site (similar

8 We here only discuss DOTBs with nonzero oscillations at 'heavy' sites as $C \rightarrow 0$. There are also solutions with nonzero codes only at 'light' sites, e.g. $\{\uparrow(\mathbb{O} 0)\}_{S}^{O}$, which can be continued down to the linear spectrum. 

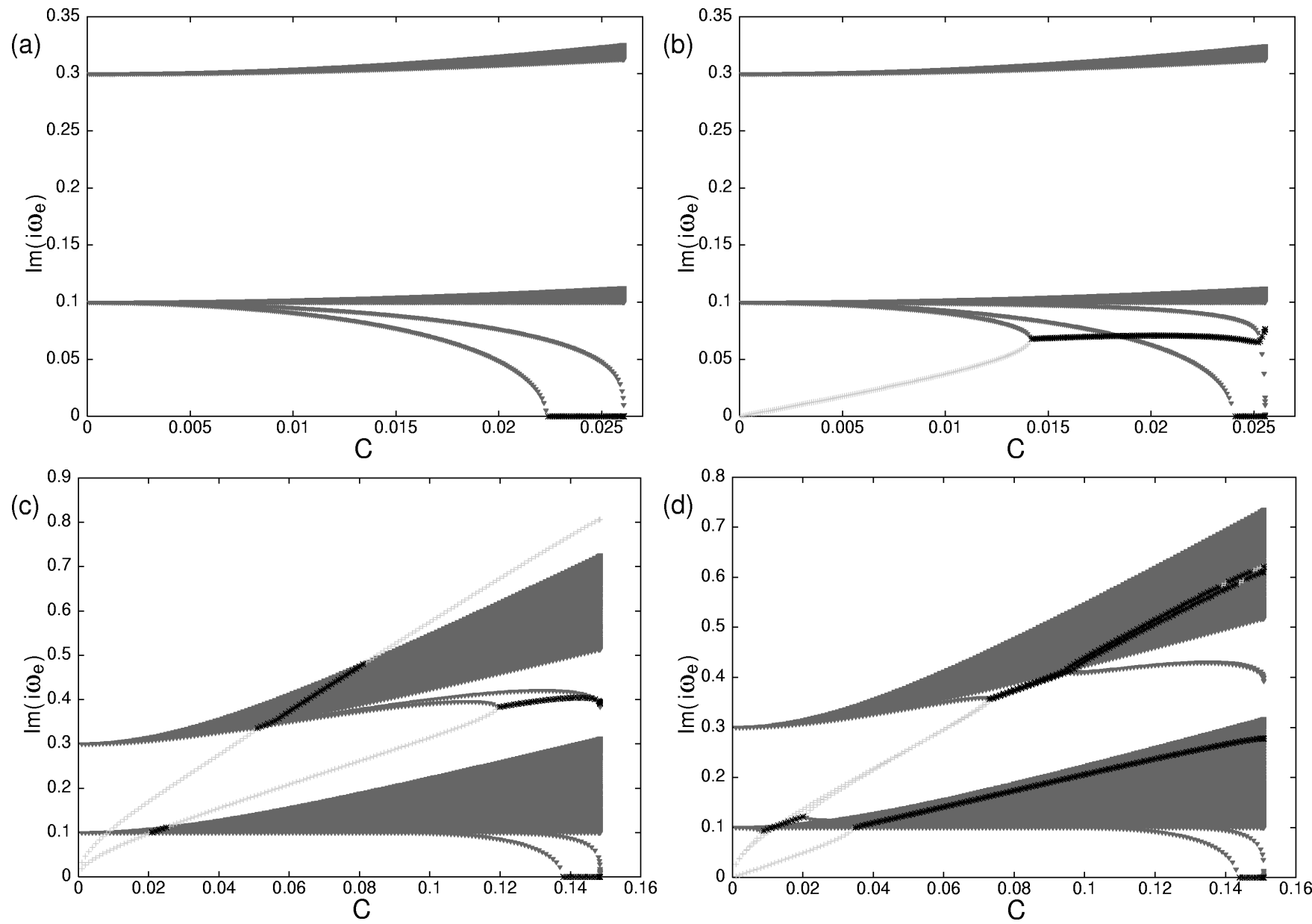

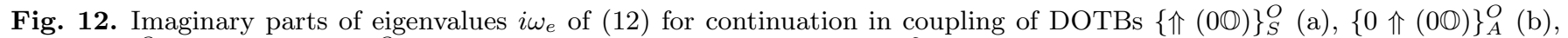
$\{\Uparrow \uparrow(\mathbb{O} 0)\}_{S}^{O}(\mathrm{c}),\{0 \Uparrow \uparrow(\mathbb{O} 0)\}_{A}^{O}(\mathrm{~d})$ at frequency deviation (19) $\Delta \omega=\delta^{2}$ (note, that $q=\pi$ for DOTBs). Light-grey crosses (dark-grey triangles) correspond to eigenvalues with $\kappa=+1(\kappa=-1)$, and black stars correspond to unstable eigenvalues.

to the $N 0$ mode). This results in an oscillatory instability of antisymmetric DOTBs (Fig. 12(b), (d)). The DOTBs $\{\Uparrow \uparrow(\mathbb{O} 0)\}_{S}^{O}$ and $\{0 \Uparrow \uparrow(\mathbb{O} 0)\}_{A}^{O}$ have two additional localized modes with $\kappa>0$, similar to the $P 1$ and $P 2$ modes described in Sec. 5.4.1 Penetrating the extended bands or colliding with other localized modes (with $\kappa<0$ ) they also produce oscillatory instabilities (Fig. 12(c), (d)).

\section{Conclusions}

We have investigated the linear stability properties of discrete gap and out-gap breathers in the modulated DNLS model, which in particular describes electromagnetic wave propagation in an array of weakly coupled optical waveguides of different widths. The coupling constant was varied from $C=0$ (anti-continuous limit) up to values, at which the localization length of the breather becomes much larger than the array constant (continuous limit).

Different types of oscillatory and real instabilities of DGBs were revealed, similar to those described for DGBs in the diatomic Klein-Gordon model 17. However, in contrast to the KG case, DGBs in infinite DNLS chains do not possess any oscillatory instability when the breather frequency is in the lower half of the gap.

The transition from gap to out-gap breathers was studied by varying the breather frequency at different fixed values of the coupling. Such transitions were found to occur in different ways, depending on the value of the coupling, and a relation to the transition from discrete- to continuous-like breathers (described earlier for DGBs in the diatomic KG model [17]) was revealed.

Discrete out-gap breathers with tails of different wave numbers were investigated, and a general bifurcation picture described. DOGBs were shown to, generally, possess the same types of oscillatory and real instabilities as DGBs. Still, there are important differences between stability properties of DGBs and DOGBs. Unlike antisymmetric DGBs, antisymmetric DOGBs are linearly stable for small coupling, so there is an interval in coupling $\left[0, C_{0}\right]$ where symmetric and antisymmetric DOGBs are stable simultaneously. Also, since DOGBs generally are non-localized, they possess strong oscillatory instabilities associated with resonances between extended lattice modes 
of the upper- and lower-band sub-fields. For DGBs such instabilities do not survive in the limit of infinite chain.

The process of breather frequency penetration into the linear spectrum and bifurcation of a DGB into a DOGB was found to have many qualitative similarities with the breather second harmonic penetration into the linear spectrum and formation of 'phonobreathers' with non-zero tails, discussed for a monoatomic KG chain in 39. However, we found no analogue to 'phantom' breathers with vanishing tails, since all DOGBs with tails of different wave numbers apparently are non-localized at frequencies inside the linear spectrum. The question about the existence of such localized excitations inside the linear wave spectrum in modulated models is thus still unresolved.

Another interesting and important question, which we address for future investigations, concerns the possibility of finding exact moving gap and out-gap breathers. As we have shown here, DGBs and DOGBs in the modulated DNLS model exhibit the 'exchange of stability' between symmetric and antisymmetric modes, and thus a considerably increased mobility of these excitations is expected for parameter values close to the stability exchange point [5]. We hope that our results will stimulate experimental activity to directly observe this phenomenon, e.g. in coupled waveguide arrays.

We recently demonstrated such DGB mobility in the diatomic KG chain [17, and connected it with a vanishing of the PN barrier. However, the determination of the PN potential, and the PN barrier as the energy difference between symmetric and antisymmetric breathers, is not unambiguous in the KG model, since for fixed values of the system parameters there exist continuous families of symmetric and antisymmetric DBs with different frequencies. In the modulated DNLS model, the existence of an additional conserved quantity, the excitation norm, makes it natural to define the $\mathrm{PN}$ barrier as the difference between energies of symmetric and antisymmetric DBs with the same norm 42. Thus it is of interest to study more in details the 'exchange of stability' in the modulated DNLS model, and to look for exact moving breathers in this regime.

Another interesting effect, which also should be experimentally observable, arises as a consequence of the existence of DGB internal modes with frequencies above the continuous spectrum (e.g. P1-P5 in Fig. 3). Since then also all multiples of the mode frequency will lie outside the continuous spectrum, similar arguments as in 43 prove the existence of exact localized quasiperiodic gap breathers, with two, generally incommensurate, frequencies. These solutions are characterized by persistent large-amplitude internal intensity oscillations. It is particularly interesting to note, that since e.g. the $P 1$ mode of the symmetric DGB is always above the continuous spectrum in the lower half of the gap when $-1.56 \lesssim \Delta \omega / \delta^{2}<-1$, quasiperiodic solutions bifurcating from this mode should exist in continuous families, arbitrarily close as well to the anticontinuous as to the continuous limit. The properties of such solutions, which cannot exist in KG models, will be discussed elsewhere.
We acknowledge support from the Royal Swedish Academy of Sciences and "Knut och Alice Wallenbergs jubileumsfond". A.V.G. acknowledges support from the Swedish Institute. M.J. acknowledges support from the Swedish Research Council.

\section{A Calculation of localized modes at small $C$}

For localized eigenmodes at small $C$, the eigenvalue problem (15) can be simplified. Indeed, at small coupling these eigenmodes are mainly localized on a few central sites (if the corresponding eigenvalues lie inside the extended band, these eigenmodes are not purely localized, but the central site amplitudes are much larger than the tails). In this case one can put $(a, b)_{n} \equiv 0$ for $\left|n-N_{c}\right| \geq N_{\epsilon}$, where $N_{\epsilon}$ characterizes the localization length of an eigenmode. Moreover, using $C$ as a small parameter, one can obtain approximate expressions for the central site amplitudes of the unperturbed solution $\phi_{n}^{(0)}$, for DGB $\{\Uparrow(0 \mathbb{O})\}_{S}^{G}$ :

$$
\begin{aligned}
& \phi_{N_{c}}^{2}=2 \delta^{2}+\Delta \omega-\frac{2}{\Delta \omega} C^{2}+\mathcal{O}\left(C^{3}\right), \\
& \phi_{N_{c} \pm 1}^{2}=\frac{2 \delta^{2}+\Delta \omega}{\Delta \omega^{2}} C^{2}+\mathcal{O}\left(C^{3}\right)
\end{aligned}
$$

$\operatorname{DGB}\{0 \Uparrow(0 \mathbb{O})\}_{A}^{G}:$

$$
\begin{aligned}
& \phi_{N_{c}}^{2} \equiv 0 \\
& \phi_{N_{c} \pm 1}^{2}=2 \delta^{2}+\Delta \omega-\frac{1}{\Delta \omega} C^{2}+\mathcal{O}\left(C^{3}\right), \\
& \phi_{N_{c} \pm 2}^{2}=\frac{2 \delta^{2}+\Delta \omega}{\Delta \omega^{2}} C^{2}+\mathcal{O}\left(C^{3}\right)
\end{aligned}
$$

$\operatorname{DOGB}\{\Uparrow(\uparrow \mathbb{O} \downarrow \mathbb{O})\}_{S}^{O}:$

$$
\begin{aligned}
& \phi_{N_{c}}^{2}=2 \delta^{2}+\Delta \omega+2 \sqrt{\frac{\Delta \omega}{2 \delta^{2}+\Delta \omega}} C+\mathcal{O}\left(C^{2}\right), \\
& \phi_{N_{c} \pm 1}^{2}=\Delta \omega+2 \sqrt{\frac{2 \delta^{2}+\Delta \omega}{\Delta \omega}} C+\mathcal{O}\left(C^{2}\right),
\end{aligned}
$$

and DOGB $\{0 \Uparrow(\uparrow \mathbb{O} \downarrow \mathbb{O})\}_{A}^{O}$ :

$$
\begin{aligned}
& \phi_{N_{c}}^{2} \equiv 0 \\
& \phi_{N_{c} \pm 1}^{2}=2 \delta^{2}+\Delta \omega+\sqrt{\frac{\Delta \omega}{2 \delta^{2}+\Delta \omega}} C+\mathcal{O}\left(C^{2}\right) \\
& \phi_{N_{c} \pm 2}^{2}=\Delta \omega+\sqrt{\frac{2 \delta^{2}+\Delta \omega}{\Delta \omega}} C+\mathcal{O}\left(C^{2}\right) .
\end{aligned}
$$

Note that $\Delta \omega<0(\Delta \omega>0)$ for gap (out-gap) breathers.

Thus, in the case of a symmetric DGB $\{\Uparrow(0 \mathbb{O})\}_{S}^{G}$, the $P 1$ mode is mainly localized on the central 'heavy' site with $n=N_{c}$, the two neighboring 'light' sites with $n=N_{c} \pm 1$, and the subsequent pair of 'heavy' sites with $n=N_{c} \pm 2$. Therefore, for small $C$ one can put $(a, b)_{n} \equiv 0$ when $\left|n-N_{c}\right| \geq 3$ in equations (15). Substituting the approximate breather solution (25) into (15) and using 
Table 1. Localized eigenmodes at small coupling. Eigenfrequencies are given at lowest significant order of $C$, with the notations: $\Delta_{1} \equiv 2 \delta^{2}+\Delta \omega$ (i.e. frequency detuning measured from the lower gap edge), $\mu_{( \pm)} \equiv\left[16 \delta^{4}+\Delta_{1}^{2} \pm \sqrt{128 \delta^{8}+\Delta_{1}^{4}}\right] /\left[4 \Delta \omega^{2} \delta^{2}\right]$. Schematic eigenvector structures are shown for the central part; higher arrows denote sites with nonzero oscillations at $C \rightarrow 0$.

\begin{tabular}{|c|c|c|c|}
\hline$\overline{\text { Eigenmode }}$ & S Schematic eigenvector structure & Eigenfrequency $\omega_{e}$ & $\Delta \overline{\Delta \omega}_{c r}$ \\
\hline$\overline{(P 1, S, G)}$ & $\ldots \mathbb{O O} 0 \Uparrow \uparrow \Downarrow \uparrow \Uparrow 0 \mathbb{O} \ldots$ & $=-\Delta \omega+2 C^{2}\left(4 \delta^{2}+\Delta \omega\right) / \Delta \omega^{2}$ & $-1.56 \cdot \delta^{2}$ \\
\hline$(P 2, S, G)$ & $\ldots \mathbb{O} 0 \Uparrow \downarrow \mathbb{O} \uparrow \Downarrow 0 \mathbb{O} \ldots$ & $\left.-\Delta \omega+C^{2}\left(4 \delta^{4}+\Delta_{1}^{2}\right) / 2 \delta^{2} \Delta \omega^{2}\right]$ & $-1.1 \cdot \delta^{2}$ \\
\hline$(P 1, A, G)$ & $\ldots \mathbb{O} 0 \Downarrow \uparrow \Downarrow \uparrow \Downarrow \uparrow \Downarrow 0 \mathbb{O} \ldots$ & $-\Delta \omega+C^{2} \mu_{(+)}$ & $-1.85 \cdot \delta^{2}$ \\
\hline$(P 2, A, G)$ & $\ldots \mathbb{O} 0 \Downarrow \uparrow \Downarrow 0 \Uparrow \downarrow \Uparrow 0 \mathbb{O} \ldots$ & $\left.-\Delta \omega+C^{2}\left(12 \delta^{4}+4 \Delta \omega \delta^{2}+\Delta \omega^{2}\right) / 2 \Delta \omega^{2} \delta^{2}\right]$ & $-1.44 \cdot \delta^{2}$ \\
\hline$(P 3, A, G)$ & $\ldots \mathbb{O} 0 \Downarrow \uparrow \Downarrow \uparrow \Downarrow \uparrow \Downarrow 0 \mathbb{O} \ldots$ & $-\Delta \omega+C^{2} \mu_{(-)}$ & $-0.86 \cdot \delta^{2}$ \\
\hline$(N 1, A, G)$ & $\ldots 0 \mathbb{O} \uparrow \uparrow \uparrow \uparrow \uparrow \mathbb{O} 0 \ldots$ & $i 2 C \sqrt{\Delta_{1}} / \sqrt{-\Delta \omega}$ & $-2 \cdot \delta^{2}$ \\
\hline$(P 1, S, O)$ & $\ldots \mathbb{O} 0 \Downarrow \downarrow \Uparrow \downarrow \Downarrow 0 \mathbb{O} \ldots$ & $\left\{6 \sqrt{\Delta \omega \Delta_{1}} C\right\}^{0.5}$ & 0 \\
\hline$(P 2, S, O)$ & $\ldots \mathbb{O} 0 \Uparrow \downarrow \mathbb{O} \uparrow \Downarrow 0 \mathbb{O} \ldots$ & $\left\{2 \sqrt{\Delta \omega \Delta_{1}} C\right\}^{0.5}$ & 0 \\
\hline$(P 1, A, O)$ & $\ldots 0 \mathbb{O} \uparrow \Downarrow 0 \Uparrow \downarrow \mathbb{O} 0 .$. & $\left\{4 \sqrt{\Delta \omega \Delta_{1}} C\right\}^{0.5}$ & 0 \\
\hline$(P 2, A, O)$ & $\ldots \mathbb{O} 0 \Uparrow \downarrow \uparrow \uparrow \Uparrow \downarrow \Uparrow 0 \mathbb{O} \ldots$ & $\left\{4 \sqrt{\Delta \omega \Delta_{1}} C\right\}^{0.5}$ & 0 \\
\hline$(P 0, A, O)$ & $\ldots \mathbb{O} 0 \Downarrow \uparrow \uparrow \uparrow \uparrow \uparrow \Downarrow 0 \mathbb{O} \ldots$ & $C \sqrt{2 \Delta_{1}^{2}-\Delta \omega^{2}} / \sqrt{\Delta \omega \Delta_{1}}$ & 0 \\
\hline$(N 0, A, O)$ & $\ldots 0 \mathbb{O} \downarrow \Uparrow \uparrow \Uparrow \downarrow \mathbb{O} 0 \ldots$ & $\Delta \omega+\mathcal{O}\left(C^{4}\right)$ & 0 \\
\hline
\end{tabular}

the symmetry of the $P 1$ mode: $(a, b)_{N_{c}-i}=(a, b)_{N_{c}+i}$, we obtain the corresponding eigenfrequency as

$$
\omega_{e}^{\{P 1, S, G\}}=-\Delta \omega+\frac{2 C^{2}}{\Delta \omega^{2}}\left(4 \delta^{2}+\Delta \omega\right)+\mathcal{O}\left(C^{3}\right) .
$$

Here the superscript marks the type of mode ( $P 1$ or $P 2)$, the symmetry of the breather $\phi_{n}^{(0)}(S[A]$ for symmetric [antisymmetric] breathers), and the type of breather $(G$ and $O$ for gap and out-gap breathers, respectively).

Analogously, for the antisymmetric P2 mode we have:

$$
\begin{aligned}
& \omega_{e}^{\{P 2, S, G\}}= \\
& \quad=-\Delta \omega+\frac{C^{2}\left(8 \delta^{4}+4 \delta^{2} \Delta \omega+\Delta \omega^{2}\right)}{2 \delta^{2} \Delta \omega^{2}}+\mathcal{O}\left(C^{3}\right) .
\end{aligned}
$$

To see if a particular eigenmode is localized or not at small $C$, i.e. whether the corresponding eigenvalue lies inside or outside the extended band, we check the difference between the eigenvalue and the band edge $\omega_{+}^{\{\max , G\}}$ (20):

$$
\omega_{+}^{\{\max , G\}}=\omega_{o}(\pi)-\omega_{b}=-\Delta \omega-\delta^{2}+\sqrt{\delta^{4}+4 C^{2}},
$$

from which it bifurcates (see Fig. 3). This yields for the $P 1$ mode

$$
\begin{aligned}
& \omega_{e}^{\{P 1, S, G\}}-\omega_{+}^{\{\max , G\}}= \\
& \quad=\frac{2 C^{2}}{\Delta \omega^{2} \delta^{2}}\left(4 \delta^{4}+\delta^{2} \Delta \omega-\Delta \omega^{2}\right)+\mathcal{O}\left(C^{3}\right) .
\end{aligned}
$$

Consequently, when the frequency detuning of a symmetric DGB is above the critical value $\Delta \omega>\Delta \omega_{c r}^{\{P 1, S, G\}} \approx$ $-1.56 \cdot \delta^{2}$, the $P 1$ eigenmode is localized at any non-zero value of $C$. By contrast, when $\Delta \omega \leq \Delta \omega_{c r}^{\{P 1, G\}}$, the $P 1$ eigenmode is not localized at small values of $C$.

Similar results are obtained for the $P 2$ mode:

$$
\begin{aligned}
& \omega_{e}^{\{P 2, S, G\}}-\omega_{+}^{\{\max , G\}}= \\
& \quad=\frac{C^{2}}{2 \Delta \omega^{2} \delta^{2}}\left(8 \delta^{4}+4 \delta^{2} \Delta \omega-3 \Delta \omega^{2}\right)+\mathcal{O}\left(C^{3}\right) .
\end{aligned}
$$

The critical value of the frequency detuning for this mode is equal to $\Delta \omega_{c r}^{\{P 2, S, G\}} \approx-1.1 \cdot \delta^{2}$.

Using the same technique, expressions for the eigenvalues and eigenvectors corresponding to localized modes at small coupling can be obtained for antisymmetric DGB $\{0 \Uparrow(0 \mathbb{O})\}_{A}^{G}$, and for out-gap breathers $\{\Uparrow(\uparrow \mathbb{O} \downarrow \mathbb{O})\}_{S}^{O}$, $\{0 \Uparrow(\uparrow \mathbb{O} \downarrow \mathbb{O})\}_{A}^{O}$. The results are presented in Table 1 where for each eigenmode the schematic eigenvector and eigenvalue are given at the lowest significant order of $C$, together with the approximate value of the critical frequency detuning $\Delta \omega_{c r}$, above which the mode is localized at small $C$. Note in particular that the antisymmetric DGB $\{0 \Uparrow(0 \mathbb{O})\}_{A}^{G}$ has an imaginary eigenfrequency corresponding to the $N 1$ mode, and thus it is unstable for small $C$. All other eigenfrequencies are real, proving the linear stability of the other three solutions for small $C$.

\section{References}

1. S. Aubry, Physica D 103, 201 (1997).

2. S. Flach, C.R. Willis, Phys. Rep. 295, 181 (1998).

3. G.P. Tsironis, S. Aubry, Phys. Rev. Lett. 77, 5225 (1996).

4. D. Chen, S. Aubry, G.P. Tsironis, Phys. Rev. Lett. 77, 4776 (1996).

5. T. Cretegny, Ph.D. thesis, École Normale Supérieure de Lyon, France, 1998 (in French).

6. W. Chen, D.L. Mills, Phys. Rev. Lett. 58, 160 (1987).

7. O.A. Chubykalo, Yu.S. Kivshar, Phys. Rev. E 48, 4128 (1993); 49, 5906(E) (1994).

8. S.A. Kiselev, S.R. Bickham, A.J. Sievers, Phys. Rev. B 48, 13508 (1993).

9. M. Aoki, S. Takeno, A.J. Sievers, J. Phys. Soc. Jpn 62, 4295 (1993).

10. S.A. Kiselev, S.R. Bickham, A.J. Sievers, Phys. Rev. B 50, 9135 (1994).

11. A. Franchini, V. Bortolani, R.F. Wallis, Phys. Rev. B 53, 5420 (1996).

12. R. Livi, M. Spicci, R.S. MacKay, Nonlinearity 10, 1421 (1997). 
13. G. James, P. Noble (submitted to Physica D).

14. T. Cretegny, R. Livi, M. Spicci, Physica D 119, 88 (1998).

15. A.V. Zolotaryuk, P. Maniadis, G.P. Tsironis, Physica B 296, 251 (2001).

16. P. Maniadis, A.V. Zolotaryuk, G.P. Tsironis, Phys. Rev. E 67, 046612 (2003).

17. A.V. Gorbach, M. Johansson, Phys. Rev. E 67, 066608 (2003).

18. A.A. Sukhorukov, Yu.S. Kivshar, H.S. Eisenberg, Y. Silberberg, IEEE J. Quantum Electron. 39, 31 (2003), and references therein.

19. A.A. Sukhorukov, Yu.S. Kivshar, Opt. Lett. 27, 2112 (2002).

20. A.A. Sukhorukov, Yu.S. Kivshar, arXiv:nlin.PS/0303054 (2003).

21. A.A. Sukhorukov, Yu.S. Kivshar, Phys. Rev. Lett. 91, 113902 (2003).

22. P.G. Kevrekidis, B.A. Malomed, Z. Musslimani, Eur. Phys. J. D 23, 421 (2003).

23. A. Trombettoni, A. Smerzi, Phys. Rev. Lett. 86, 2353 (2001); A. Smerzi, A. Trombettoni, Chaos 13, 766 (2003).

24. F.S. Cataliotti et. al., Science 293, 843 (2001).

25. J.C. Eilbeck, M. Johansson, in "Localization and Energy Transfer in Nonlinear Systems", edited by L. Vázquez, M.P. Zorzano, R. MacKay (World Scientific, Singapore, in press); arXiv: nlin.PS/0211049 (2002).

26. Yu.S. Kivshar, M. Peyrard, Phys. Rev. A 46, 3198 (1992); Yu.S. Kivshar, Phys. Lett. A 173, 172 (1993); I. Daumont, T. Dauxois, M. Peyrard, Nonlinearity 10, 617 (1997).

27. A.M. Morgante, M. Johansson, G. Kopidakis, S. Aubry, Physica D 162, 53 (2002).

28. Yu.S. Kivshar and N. Flytzanis, Phys. Rev. A 46, 7972 (1992).

29. A.S. Kovalev, O.V. Usatenko, A.V. Gorbatch, Phys. Rev. E 60, 2309 (1999).

30. J. Coste, J. Peyraud, Phys. Rev. B 39, 13086 (1989); 39, 13096 (1989).

31. A.V. Yulin, D.V. Skryabin, Phys. Rev. A 67, 023611 (2003).

32. J. Yang, B.A. Malomed, D.J. Kaup, A.R. Champneys, Math. Comput. Simul. 56, 585 (2001).

33. A. Franchini, V. Bortolani, R.F. Wallis, J. Phys.: Condens. Matter 14, 145 (2002).

34. J. Carr, J.C. Eilbeck, Phys. Lett. A 109, 201 (1985).

35. T.J. Bridges, Proc. R. Soc. Lond. A 453, 1365 (1997).

36. D.V. Skryabin, J. Opt. Soc. Am. B 19, 529 (2002).

37. J.L. Marín, S. Aubry, Nonlinearity 9, 1501 (1996).

38. J.C. Eilbeck, P.S. Lomdahl, A.C. Scott, Phys. Rev. B 30, 4703 (1984); Physica D 16, 318 (1985).

39. A.M. Morgante, M. Johansson, S. Aubry, G. Kopidakis, J. Phys. A: Math. Gen. 35, 4999 (2002).

40. J.L Marín, S Aubry, Physica D 119, 163 (1998).

41. N.G Vakhitov, A.A Kolokolov Radiophys Quantum Electron 16, 783 (1975). [Translated from Izvestiya Vysshikh Uchebnykh Zavedenii, Radiofizika 16, 1020 (1973).]

42. Yu.S. Kivshar and D.K. Campbell, Phys. Rev. E 48, 3077 (1993).

43. P.G. Kevrekidis and M.I. Weinstein, Math. Comput. Simul. 62, 65 (2003). 